\title{
17. OXYGEN AND CARBON ISOTOPE STRATIGRAPHY OF THE MIDDLE MIOCENE, HOLES 805B AND 806B ${ }^{1}$
}

\author{
Richard M. Corfield ${ }^{2}$ and Julie E. Cartlidge ${ }^{2}$
}

\begin{abstract}
Bulk carbon isotope records are an effective chemostratigraphic tool for the middle Miocene because of the large and systematic variation in first-order $\delta^{13} \mathrm{C}$ signals. Bulk $\delta^{13} \mathrm{C}$ measurements support the presence of a hiatus at $305 \mathrm{mbsf}$ in Hole 805B (latest middle Miocene), provisionally located while on board ship using biostratigraphic and magnetostratigraphic events. Records at Holes 805B and 806B show the middle Miocene Monterey carbon isotope excursion although the record at Hole 806B is apparently more stratigraphically continuous. Detailed analysis of multispecies foraminiferal carbon isotope records during the middle Miocene ("Monterey excursion") segment at Hole 806B support the assertion that this carbon isotope excursion comprises mainly between-reservoir effects. The benthic $\delta^{18} \mathrm{O}$ data increase after $15.3 \mathrm{Ma}$, which we suggest corresponds to the mid-Miocene cooling step/ice volume increase of other authors. Planktonic foraminiferal $\delta^{18} \mathrm{O}$ evidence exists for steepening of the thermocline at 17.4 Ma. A second-order $\delta^{13} \mathrm{C}$ excursion superimposed at $13.8 \mathrm{Ma}$ on the first-order Monterey excursion is associated with a second-order negative $\delta^{18} \mathrm{O}$ excursion.
\end{abstract}

\section{INTRODUCTION}

One of the most important achievements of Ocean Drilling Program (ODP) Leg 130 was the recovery of thick middle Miocene carbonate oozes and chalks drilled using the advanced hydraulic piston corer (APC), especially at Holes 805B and 806B (Fig. 1). Sedimentation rates at these sites were relatively high $(12-13 \mathrm{~m} / \mathrm{m}$.y. at Hole $805 \mathrm{~B}$ and between 20 and $30 \mathrm{~m} / \mathrm{m} . \mathrm{y}$. at Hole 806B, as documented by the Shipboard Scientific Party), and this permits the generation of high-resolution $\delta^{18} \mathrm{O}$ and $\delta^{13} \mathrm{C}$ records from the western equatorial Pacific through the middle Miocene. From the perspective of $\delta^{18} \mathrm{O}$ reconstruction of marine paleoclimates, this is an important region because the thermal stability of the surface waters (Matthews and Poore, 1980; Prentice and Matthews, 1988; Ravelo et al., 1990) may permit further constraints on the relative contribution of icevolume and temperature change to the marine carbonate $\delta^{18} \mathrm{O}$ signal (Miller et al., 1991).

Several previous studies have focused on this important interval at other sites. Shackleton and Kennett (1975) and Savin et al. (1975) were the first to identify the profound $\delta^{18} \mathrm{O}$ increase in benthic foraminifers that has been associated with the onset of Antarctic glaciation (Shackleton and Kennett, 1975; Savin et al., 1975, 1981; Woodruff et al., 1981), although the significance of this change in terms of ice-volume vs. temperature contribution to the $\delta^{18} \mathrm{O}$ signal remains obscure (Matthews and Poore, 1980; see discussion in Miller et al., 1991). Woodruff et al. (1981) analyzed the benthic $\delta^{18} \mathrm{O}$ record in detail at Deep Sea Drilling Project (DSDP) Site 289 (close to Hole 806B; Fig. 1) and concluded that the onset of major Antarctic glaciation occurred between 14.8 and 14.0 Ma. Shackleton (1982) examined the planktonic record at Site 289 and concluded that the middle Miocene stable isotope record offered the potential to examine climatic variability on time scales similar to that at present possible in the Pleistocene.

In a more recent series of studies, several authors (Savin et al., 1985; Barrera et al., 1985; Vincent et al., 1985; Miller et al., 1989, 1991; Wright et al., 1992) have identified this mid-Miocene ${ }^{18} \mathrm{O}$ enrichment in deep waters at a number of sites in the Atlantic, Indian,

\footnotetext{
'Berger, W.H., Kroenke, L.W., Mayer, L.A., et al., 1993. Proc. ODP, Sci. Resuits, 130: College Station, TX (Ocean Drilling Program).

2 Department of Earth Sciences, University of Oxford, Park's Road, Oxford OX1 3PR, United Kingdom.
}

and Pacific oceans. However, estimates of the precise timing of this cooling episode vary. Woodruff and Savin (1989) developed a model that identifies a profound change in the nature of deep-water circulation during this interval. They hypothesize that the early Miocene was a time when deep-water circulation was dominated by Tethyan outflow but that following the mid-Miocene ${ }^{18} \mathrm{O}$ enrichment the emphasis shifted to the production of cool, dense waters in the Antarctic region. Wright et al. (1992) used $\delta^{13} \mathrm{C}$ variations in benthic foraminifers to monitor deep-water movements during the early and middle Miocene and concluded that two modes of deep-water formation were present during this interval. Between 24-20 Ma and 16-12.5 Ma, the deep oceans were ventilated by Southern Component Water (SCW), which is analogous to Antarctic Bottom Water (AABW); however, during other intervals, deep-water formation was from multiple sources with relatively warm Northern Component Water (NCW; analogous to North Atlantic Deep Water [NADW]) and Tethyan water masses (as suggested by Woodruff and Savin, 1989), supplementing SCW production between 20 and $16 \mathrm{Ma}$ and NCW and SCW production dominating between 12.5 and $10 \mathrm{Ma}$. The combination of NCW and Tethyan water mass production between 20 and $16 \mathrm{Ma}$ warmed the deep ocean by $3^{\circ}-4^{\circ}$; after $16 \mathrm{Ma}$, deep-water temperatures cooled and have remained relatively cold ever since.

Stratigraphic reference sections for the middle Miocene interval based on stable isotopic changes in foraminifers have also been established. Loutit et al. (1983) subdivided the middle Miocene on the basis of $\delta^{13} \mathrm{C}$ inflections and claimed that this improved the precision of stratigraphic correlation of events to $\pm 105 \mathrm{yr}$. Miller et al. (1991) and Wright and Miller (1992) defined eight oxygen isotope zones (their "Mi" zones), based on $\delta^{18} \mathrm{O}$ inflections in benthic foraminifers that they assert correlate with glacioeustatic sea-level changes.

Because ice-volume fluctuations, temperature effects, and salinity-correlated effects influence oxygen isotope fractionation, $\delta^{18} \mathrm{O}$ records from foraminifers cannot be uniquely interpreted in terms of a single variable (see discussion in Miller et al., 1991). As a consequence of this, a considerable body of work has focused on quantifying the different contributions of these three variables to Cenozoic (particularly Pleistocene) $\delta^{18} \mathrm{O}$ records. The usual approach to estimate the ice volume/temperature effect contribution is to compare planktonic foraminiferal records from tropical or subtropical regions and benthic foraminiferal $\delta^{18} \mathrm{O}$ records. This strategy is based on the assumption that ice-volume growth or decay will affect both benthic 


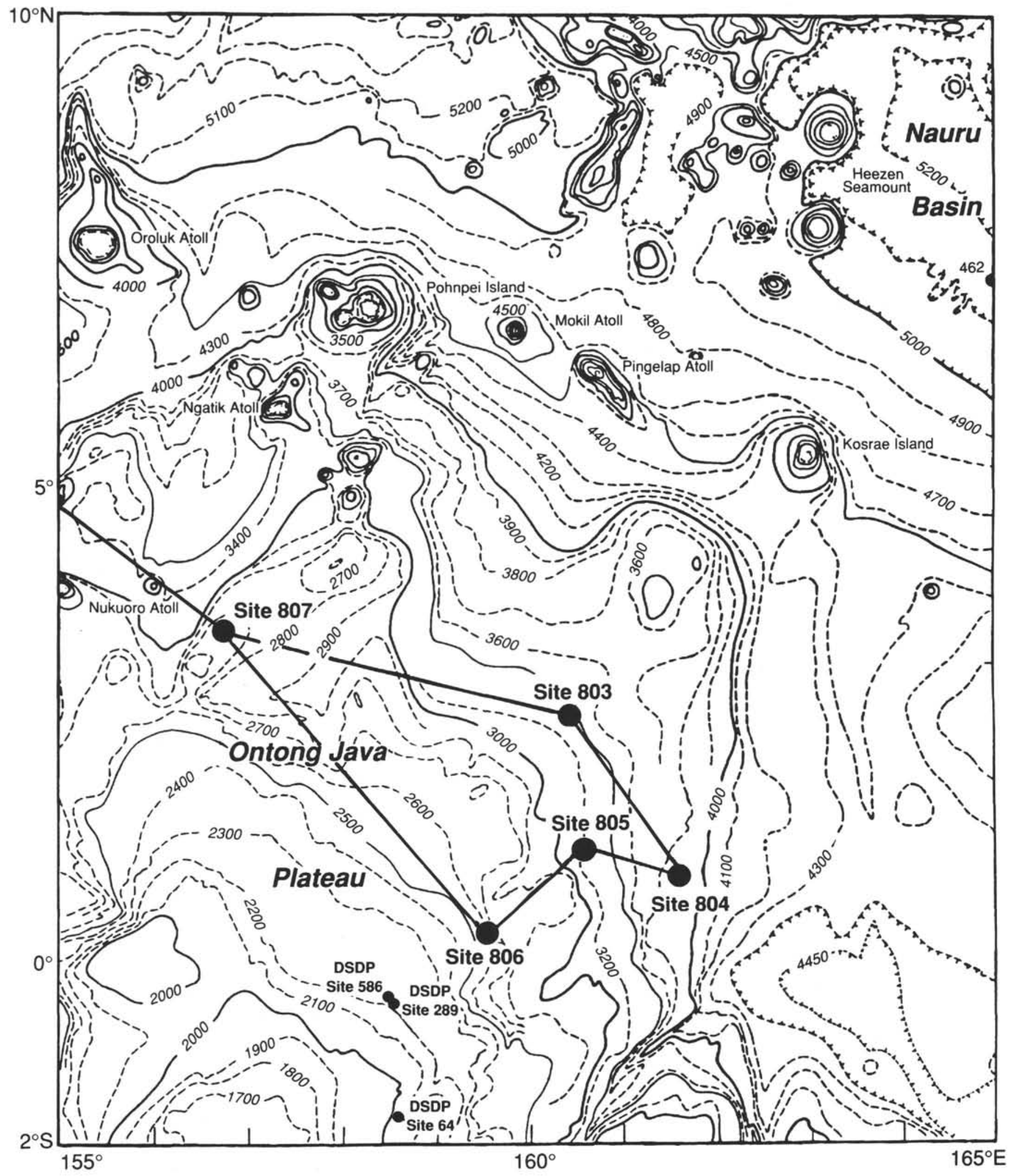

Figure 1. Location map of Leg 130 sites, including Holes 805B and 806B. 
and planktonic records whereas temperature change will register only in planktonic records (e.g., Shackleton, 1967; Shackleton and Opdyke, 1973, 1976; Fairbanks and Matthews, 1978). Miller et al. (1991) advocate a similar approach further back in the Cenozoic, but they point out that the western equatorial regions offer the opportunity to obtain $\delta^{18} \mathrm{O}$ records from planktonic foraminifers that are less likely to be affected by temperature variations than other regions because of their thermal stability (Ravelo et al., 1990).

Vincent and Berger (1985) developed a significant model that linked the middle Miocene ${ }^{18} \mathrm{O}$ enrichment in benthic $\delta^{18} \mathrm{O}$ records to the isotopically positive ${ }^{13} \mathrm{C}$ values that typify middle Miocene foraminifers. They named this positive $\delta^{13} \mathrm{C}$ interval the "Monterey excursion."

Our aim in this contribution is to develop an oxygen and carbon isotope stratigraphy for Holes $805 \mathrm{~B}$ and $806 \mathrm{~B}$ in the interval between 12 and $20 \mathrm{Ma}$. We wish to investigate the isotopically positive $\delta^{13} \mathrm{C}$ values that characterize the early to middle Miocene interval and also to investigate their possible climatic consequences in the western equatorial Pacific. In conducting this preliminary contribution, our data were collected at a sampling resolution of one per core section (Appendixes A and B). This work continues at a higher sampling resolution.

\section{METHODS}

The $\delta^{13} \mathrm{C}$ and $\delta^{18} \mathrm{O}$ stratigraphies generated by the analysis of bulk samples were used to identify discontinuities that might be caused by the presence of hiatuses. In addition, we picked up to five species of planktonic foraminifers and four species of benthic foraminifers for study. However, because our focus is on the changing vertical gradients of $\delta^{18} \mathrm{O}$ and $\delta^{13} \mathrm{C}$ across this time interval, we restrict the present discussion to data derived from a surface-water-dwelling planktonic foraminiferal species (Dentoglobigerina altispira), a deeper dwelling planktonic foraminiferal species (Globoquadrina venezuelana), and benthic foraminifers (usually Cibicidoides spp. and Planulina wuellerstorfi). The benthic foraminifer data were corrected for isotopic disequilibrium effects using the correction factors of Shackleton et al. (1984).

Foraminiferal samples were disaggregated in distilled water. Between 5 and 15 planktonic specimens were picked from the 300-355$\mu \mathrm{m}$ size fraction to minimize ontogenetic effects. The number of benthic specimens picked from this size fraction was generally lower as a result of their scarcity within the samples. Samples were cleaned using $\mathrm{H}_{2} \mathrm{O}_{2}$ and $\left(\mathrm{CH}_{3}\right)_{2} \mathrm{CO}$ and then dried for $30 \mathrm{~min}$ at $60^{\circ} \mathrm{C}$. Samples were analyzed isotopically using a VG Isotech PRISM mass spectrometer in the Oxford Laboratory calibrated to NBS 19 and Cambridge Carrara marble.

The lower portion of the interval studied at Hole 806B in particular was relatively deeply buried ( $300-600 \mathrm{mbsf})$. The interval studied at Hole $805 \mathrm{~B}$ was shallower (290-390 mbsf). In sections more deeply buried than about $400 \mathrm{~m}$, diagenetic alteration of stable isotope ratios becomes an important consideration (Miller and Curry, 1982; Barrera et al., 1987; Wright et al., 1992). Clearly, therefore, the possibility of diagenetic overprinting must be investigated in the deeper portions of Hole 806B. This is especially important because the adjacent DSDP Site 289 has a similar burial depth and has been used as a reference section for this interval in the western equatorial Pacific (Shackleton, 1982; Wright et al., 1991, 1992). Other authors have suggested that the $\delta^{18} \mathrm{O}$ signal may be degraded because of diagenetic overprinting (Elderfield et al., 1982; Miller et al., 1991). We have followed the approach used by Wright et al. (1992) and adopted two criteria for the identification of diagenesis: (1) optical examination for calcite overgrowths and (2) similarity to other, shallower records. With respect to the first criterion, sediments disaggregated easily, suggesting only minimal cementation and significant calcite overgrowth was not observed. Comparison of the $\delta^{18} \mathrm{O}$ record from Hole $806 \mathrm{~B}$ with other records is discussed below. In general, however, the major features of middle Miocene $\delta^{13} \mathrm{C}$ and $\delta^{18} \mathrm{O}$ history are well represented in Holes $805 \mathrm{~B}$ and $806 \mathrm{~B}$, and we conclude therefore that diagenetic overprinting is not a major problem.

\section{RESULTS AND DISCUSSION \\ Bulk Isotope Stratigraphy}

Figures 2 and 3 illustrate the middle Miocene bulk isotope stratigraphy at Holes $805 \mathrm{~B}$ and $806 \mathrm{~B}$. Both Figures 2 and 3 exhibit $\delta^{13} \mathrm{C}$ values that reach broad maxima in the middle of the sections studied. At Hole $805 \mathrm{~B}$, these values are most positive between Cores 130 805B-35X ( $325 \mathrm{mbsf}$ ) and -38X (352 mbsf). At Hole 806B, a particularly positive second-order $\delta^{13} \mathrm{C}$ excursion (marked " $\mathrm{A}$ " on Fig. 3) is discernible between Cores 130-806B-50X (452 mbsf) and -52X ( $470 \mathrm{mbsf}$ ), whereas values in general remain high between Cores $130-806 \mathrm{~B}-53 \mathrm{X}$ (490 mbsf) and -55X (530 mbsf). We interpret this positive first-order feature to be the local expression of the middle Miocene $\delta^{13} \mathrm{C}$ excursion documented by Vincent and Berger (1985). The second-order excursion may correlate with one of the benthic $\delta^{13} \mathrm{C}$ events of Loutit et al. (1983). Pending further examination, we have tentatively identified this as the "Zone N11 subevent."

Detailed analysis of Hole 805B suggests that the middle Miocene portion of Hole $805 \mathrm{~B}$ is less continuous than the equivalent section at Hole $806 \mathrm{~B}$. In particular, we note steps in the $\delta^{13} \mathrm{C}$ signal within Core 130-805B-33X (between Samples 130-805B-33X-3, 139-141 cm, and $-33 X-4,9-11 \mathrm{~cm}$; marked "A" on Fig. 2) that correspond to a hiatus as identified by the shipboard biostratigraphers. Breaks in sedimentation may exist between Samples 130-805B-35X-3, 99-101 cm, and $-35 X-4,9-11 \mathrm{~cm}$ (marked "B" on Fig. 2) and between Samples 130-805B-37X-4, 69-71 cm, and -38X-1, 59-61 cm (marked "C" on Fig. 2). Judging from the positive $\delta^{13} \mathrm{C}$ values immediately above our suggested stratigraphic break at point " $\mathrm{B}$," we suggest that the secondorder $\delta^{13} \mathrm{C}$ excursion, which we have termed the "Zone N11 subevent" (marked "A" on Fig. 3), is probably partially missing in Hole 805B.

It is clear that the bulk carbonate records of the first-order midMiocene $\delta^{13} \mathrm{C}$ excursion provide a sufficiently systematic signal of high signal-to-noise ratio to serve as a high-resolution stratigraphic tool. In this it is comparable to the large amplitude of the $\delta^{13} \mathrm{C}$ signal in the Paleocene, which similarly provides high-resolution chemostratigraphic control as discussed by Shackleton et al. (1985).

In contrast, the bulk $\delta^{18} \mathrm{O}$ records at Holes $805 \mathrm{~B}$ and $806 \mathrm{~B}$ do not show systematic change over the whole of the middle Miocene interval. However, systematic changes on shorter time scales are visible. Hole $805 \mathrm{~B}$ shows a trend toward positive $\delta^{18} \mathrm{O}$ values between Cores 130-805B-34X and $-33 \mathrm{X}$ (marked "D" on Fig. 2), and Hole 806B shows the same trend between Core 130-805B-48X and -45X (marked "B" on Fig. 3). This probably corresponds to the ${ }^{18} \mathrm{O}$ enrichment associated with the cooling/ice-volume increase effect discussed in the introduction and well known from the benthic records at several sites (e.g., Miller et al., 1991). In general, however, the greater amplitude of the high-frequency variation in the bulk carbonate $\delta^{18} \mathrm{O}$ signal in the middle Miocene sequence at Holes $805 \mathrm{~B}$ and $806 \mathrm{~B}$ probably precludes the same stratigraphic usefulness as the $\delta^{13} \mathrm{C}$ signal.

A possible exception to this is the pronounced positive excursion between Cores 130-806B-50X (452 mbsf) and -52X (470 mbsf) (marked " $\mathrm{C}$ " in Fig. 3), which is contemporaneous with the maximum noted in the d $13 \mathrm{C}$ record. These positive $\delta^{1.8} \mathrm{O}$ values are missing from Hole $805 \mathrm{~B}$, which supports our assertion that this portion of the succession is missing in Hole 805B.

Based on our assessment of the stratigraphic continuity of Holes $805 \mathrm{~B}$ and $806 \mathrm{~B}$, we have concentrated this initial investigation on the apparently more complete record of Hole $806 \mathrm{~B}$. We intend to combine records from Holes $805 \mathrm{~B}$ and $806 \mathrm{~B}$ as our contribution to the highresolution Neogene stratigraphy project of the Leg 130 Shipboard Scientific Party. 


\begin{tabular}{|c|c|c|}
\hline 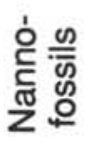 & 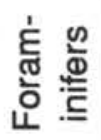 & ঠั \\
\hline NN9? & N14 & $31 x$ \\
\hline \multirow{2}{*}{$\begin{array}{l}\text { NN7. } \\
\text { NN6 }\end{array}$} & \multirow[b]{2}{*}{ N12 } & $32 x$ \\
\hline & & $33 x$ \\
\hline \multirow[t]{2}{*}{ NN6 } & N12- & $34 x$ \\
\hline & & \multirow{2}{*}{$35 x$} \\
\hline \multirow{8}{*}{ NN5 } & N11 & \\
\hline & & $36 x$ \\
\hline & Nio & $37 X$ \\
\hline & $\begin{array}{l}\text { N10- } \\
\text { N9 }\end{array}$ & \\
\hline & & SOג \\
\hline & N9 & $39 x$ \\
\hline & & $40 x$ \\
\hline & $\begin{array}{l}\text { N9- } \\
\text { N8 }\end{array}$ & \\
\hline NN5- & N7- & $41 X$ \\
\hline NN4 & N6 & $42 x$ \\
\hline
\end{tabular}
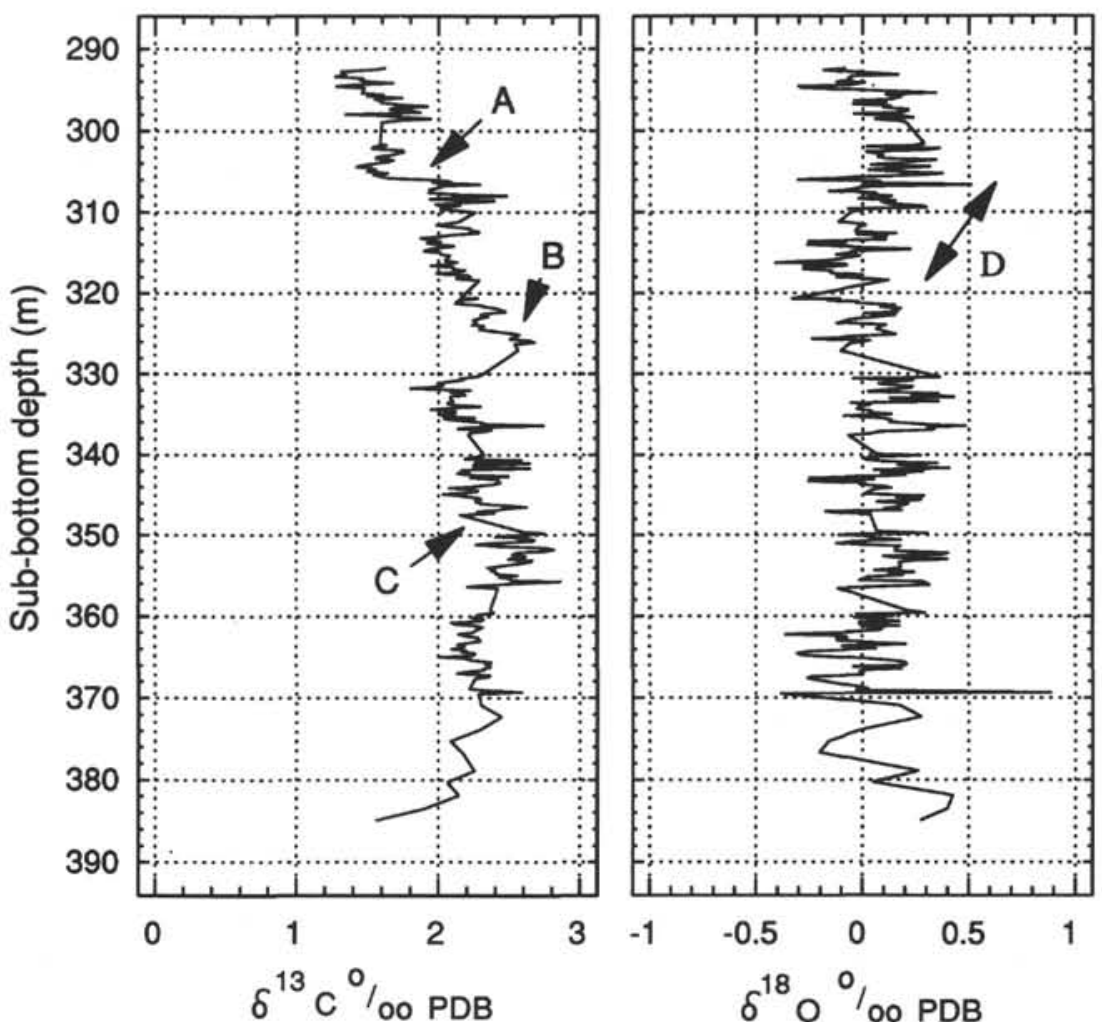

Figure 2. Bulk sample oxygen and carbon isotope stratigraphy, Hole 805B. $\mathrm{A}=$ probable hiatus in succession also noted by shipboard bio- and magnetostratigraphers, $\mathrm{B}$ and $\mathrm{C}=$ possible hiatuses within Monterey carbon isotope excursion, $\mathrm{D}=$ positive $\delta^{18} \mathrm{O}$ excursion.

\section{Foraminiferal Isotope Stratigraphy}

Figure 4 illustrates the data set for Hole 806B. The Monterey $\delta^{13} \mathrm{C}$ excursion is clearly expressed in all three groups of foraminifers. In particular, the second-order positive excursion between Cores 130806B-50X ( $452 \mathrm{mbsf}$ ) and $-52 \mathrm{X}$ is clearly depicted in D. altispira, G. venezuelana, and the benthic foraminifers. Examination of the $\delta^{18} \mathrm{O}$ data shows that this second-order event is contemporaneous with a marked positive excursion in both sub-surface dwellers ( $G$. venezuelana) and benthic foraminifers.

The $\delta^{18} \mathrm{O}$ data for all three groups of foraminifers trend toward negative values between 600 and $550 \mathrm{mbsf}$, which we interpret as the early Miocene warming trend noted by Vincent and Berger (1985). Within Core 130-806B-58X ( $550 \mathrm{mbsf}$ ), separation of the $D$. altispira and $G$. venezuelana $\delta^{18} \mathrm{O}$ signals occurs. The $\delta^{13} \mathrm{C}$ signals for the same species separate at about the same level, and we interpret this as the steepening of the thermocline and probably the sub-surface oxygen minimum as suggested by Vincent and Berger (1985). Within Core $130-806 \mathrm{~B}-54 \mathrm{X}$, benthic $\delta^{18} \mathrm{O}$ values start to trend toward more positive values, which we interpret as the cooling/ice-volume increase associated with the buildup of Antarctic ice.

We developed a chronology for our samples from Hole 806B using the biostratigraphic datums identified by Takayama (this volume). We used the time scale of Berggren et al. (1985) and calculated sample ages by linear interpolation between age control points. To reduce scatter in the data over this relatively long interval, we stacked the data into 0.1-m.y. increments of modeled time. Figure 5 shows the bulk data when processed by this method, and Figure 6 illustrates the foraminiferal data. A problem with Hole 806B is the lack of detailed magnetostratigraphy. Hence, our chronology, which is based solely at present on the nannofossil zonation of Takayama (this volume), must be regarded as preliminary.

Figure 5 shows clearly the start of the Monterey excursion at $18.75 \mathrm{Ma}$. Values increase to $16.8 \mathrm{Ma}$ and then stabilize at about $2.3 \%$ before declining again at $14.6 \mathrm{Ma}$. Subsequently, $\delta^{13} \mathrm{C}$ values peak to values of $2.7 \%$ at $13.8 \mathrm{Ma}$. This is the expression in the bulk sediment of our foraminiferal "Zone $\mathrm{N} 11$ subevent." The $\delta^{18} \mathrm{O}$ values remain rather constant throughout the succession with the exception of a positive inflection between 14.3 and $13.4 \mathrm{Ma}$, which is contemporaneous with the "Zone N11 subevent."

Figure 6 illustrates the foraminiferal data chronologically. The possible steepening of the thermocline discussed previously occurs at $17.4 \mathrm{Ma}$. Our data suggest that the onset of the increasing $\delta^{13} \mathrm{C}$ values characteristic of the Monterey excursion occur in planktonic foraminifers at about $18.8 \mathrm{Ma}$. This is effectively contemporaneous with the onset of the increase in bulk sediment $\delta^{13} \mathrm{C}$ values. However, our benthic $\delta^{13} \mathrm{C}$ data do not start to increase until about $17.9 \mathrm{Ma}$; this lag is likely to be an artifact our low benthic sampling density.

Vincent and Berger (1985) inferred on the basis of covarying foraminiferal $\delta^{13} \mathrm{C}$ data from DSDP Site 216 that the middle Miocene carbon isotope maximum was composed largely of between-reservoir carbon isotope fractionation effects. In contrast, Corfield and Cartlidge (1992) noted that the Paleocene carbon isotope maximum was comprised of both between-reservoir (burial of organic carbon) and within-reservoir (surface-water productivity) change. We have assessed the intencity of the vertical carbon isotope gradient during the 

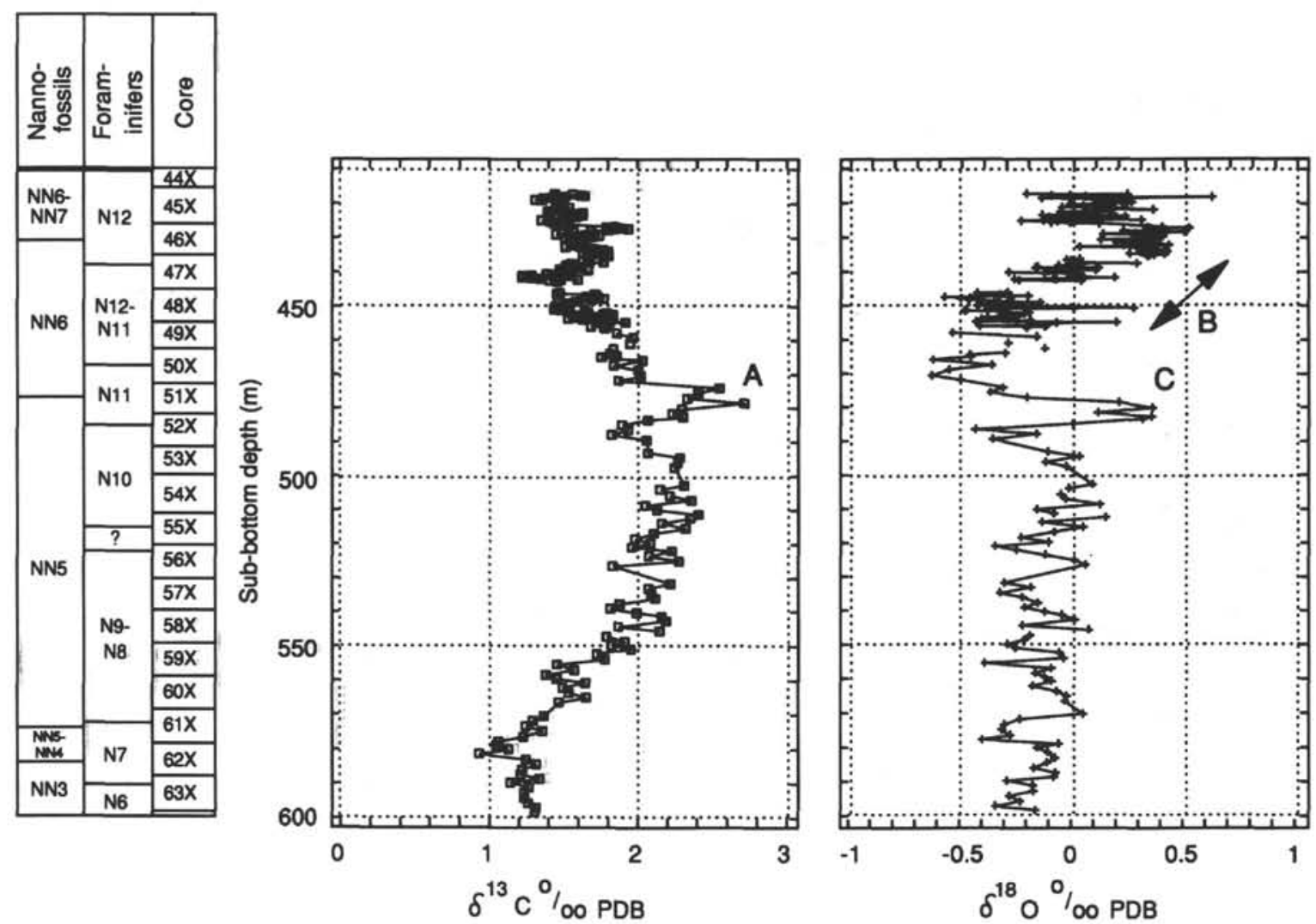

Figure 3. Bulk sample oxygen and carbon isotope stratigraphy, Hole $806 \mathrm{~B} . \mathrm{A}=$ "Zone $\mathrm{N} 11 \delta^{13} \mathrm{C}$ subevent" discussed in the text, $\mathrm{B}=$ positive $\delta^{18} \mathrm{O}$ excursion, $\mathrm{C}=$ "Zone $\mathrm{N} 11 \delta^{18} \mathrm{O}$ subevent" discussed in the text.

middle Miocene interval at Hole $806 \mathrm{~B}\left(\Delta \delta^{13} \mathrm{C}\right)$ by subtracting benthic $\delta^{13} \mathrm{C}$ values from $D$. altispira values $\left(\delta^{13} \mathrm{C}_{\text {alt-ben }}\right)$ as well as subtracting $G$. venezuelana values from $D$. altispira $\left(\delta^{13} C_{\text {alt-ven }}\right)$ values. The results are plotted as a function of bulk $\delta^{13} \mathrm{C}$ (Fig. 7). This plot suggests a weak increase in $\Delta \delta^{13} \mathrm{C}_{\text {alt-ven }}$, whereas no relationship exists between bulk $\delta^{13} \mathrm{C}$ values and $\Delta \delta^{13} \mathrm{C}_{\text {alt-ben. }}$. This suggests that a minor increase in surface-water productivity may have been associated with the Monterey excursion but that the bulk of the increase in $\delta^{13} \mathrm{C}$ values was caused by the lithospheric sequestration of isotopically light carbon. This finding is consistent with the hypothesis of Vincent and Berger (1985) that the Monterey carbon isotope excursion was caused by an increase in the rate of burial of organic carbon.

Vincent and Berger (1985) and Berger and Vincent (1986) related the middle Miocene increase in benthic $\delta^{18} \mathrm{O}$ values found globally to the increase in $\delta^{13} \mathrm{C}$ values in surface, deeper water, and benthic foraminifers. The mechanism they propose is that an increase in the rate of burial of organic carbon in the sediments rimming the Pacific Basin (the Monterey Shale and time-equivalent organic-rich strata) cause re-equilibration of $\mathrm{CO}_{2}$ between the ocean and the atmosphere, with the result that atmospheric $\mathrm{pCO}_{2}$ decreased and triggered polar cooling. Our data broadly support their speculation although we note that explicitly quantitative modeling experiments are required to verify their hypothesis. Our estimates of the chronology (subject to the reservations outlined above) of these events are as follows:

1. The $\delta^{13} \mathrm{C}$ values in bulk sediment, as well as surface and deeper dwelling planktonic foraminifers, begin to increase at $18.8 \mathrm{Ma}$. The $\delta^{13} \mathrm{C}$ increase in benthic foraminifers begins at $17.9 \mathrm{Ma}$; however, this lag may be a function of our low benthic sampling density. The increase in $\delta^{13} \mathrm{C}$ in these three groups of foraminifers may indicate the onset of organic carbon burial in Pacific Basin sediments.

2. The $\delta^{18} \mathrm{O}$ records of surface and deeper dwelling planktonic foraminifers begin to diverge at $17.4 \mathrm{Ma}$, suggesting a steepening in the thermocline.

3. The benthic $\delta^{18} \mathrm{O}$ record begins to increase at $15.3 \mathrm{Ma}$.

4. By the early/middle Miocene boundary (16.4 Ma), the thermocline is at its steepest in our record and the broad maximum of the Monterey carbon excursion has been reached.

5. The "Zone N11 subevent" occurs between 14.1 and 13.2 Ma. The $\delta^{13} \mathrm{C}$ values peak to their most positive Miocene values in all three foraminiferal groups. Contemporaneous deeper dwelling planktonic and benthic $\delta^{18} \mathrm{O}$ values increase and then recover. This positive $\delta^{18} \mathrm{O}$ inflection in the benthic curve may be the expression in the record from Hole 806B of oxygen isotope Zone Mi3 of Miller et al. (1991). The $\delta^{13} \mathrm{C}$ excursion with which it is apparently associated has been identified in other sites from benthic $\delta^{13} \mathrm{C}$ data (e.g., DSDP Sites 289 and 563; see fig. 14 in Wright et al., 1992), although this $\delta^{13} \mathrm{C}$ event has apparently not been explicitly named. Our data from Hole 806B suggests that our "Zone N11 subevent" affects all foraminiferal groups examined in this study and therefore all levels in the water column. If, as Miller et al. (1991) assert, oxygen isotope Zone Mi3 is associated with glacioeustatic lowering, this is not surprising. However, the reasons behind the apparently synchronous $\delta^{18} \mathrm{O}$ and $\delta^{13} \mathrm{C}$ change during this event need further examination.

Finally, our planktonic and benthic foraminiferal data from this western equatorial site may contribute some additional constraints to the discussion concerning the relative contributions of ice-volume 

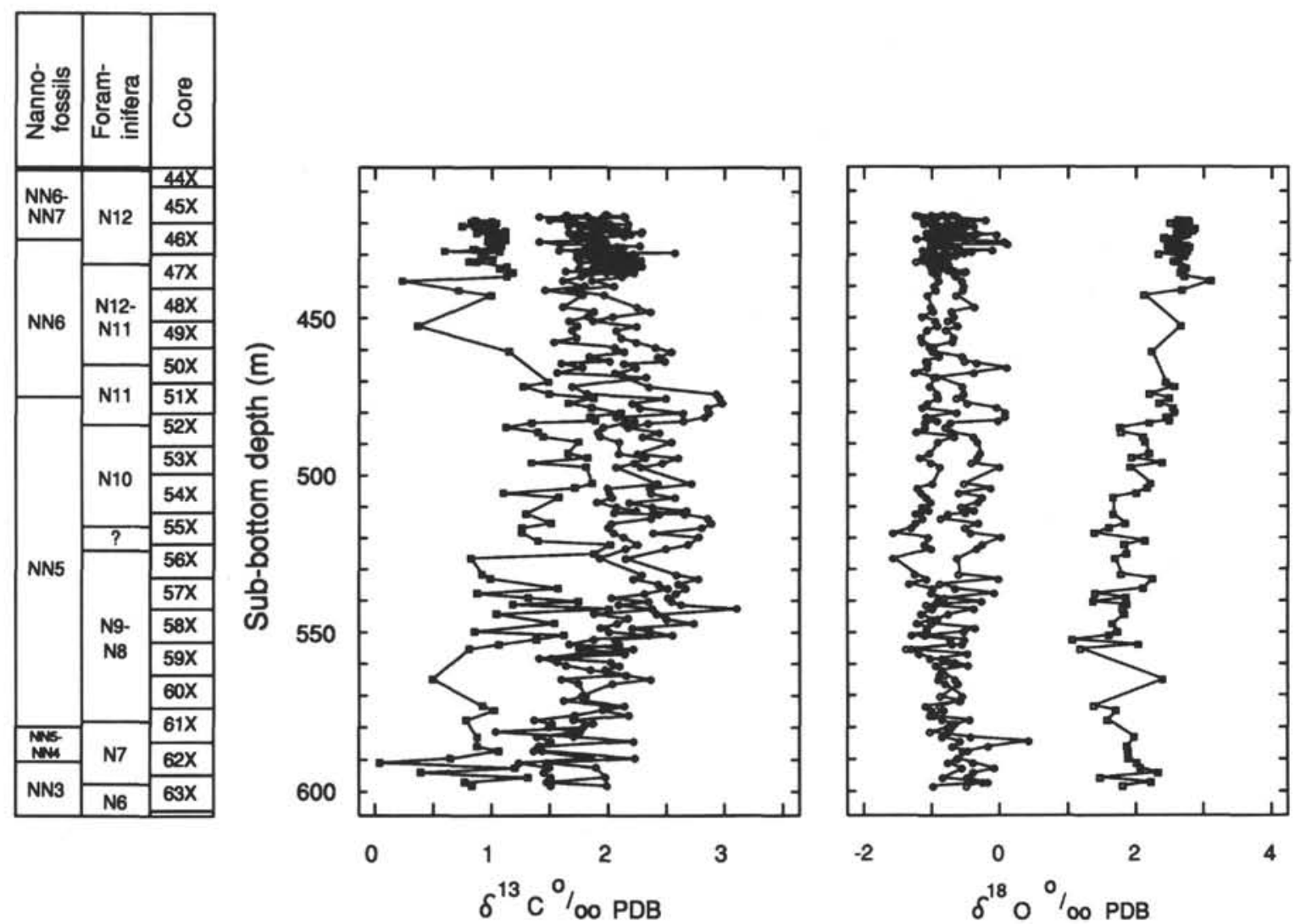

Figure 4. Foraminiferal $\delta^{13} \mathrm{C}$ and $\delta^{18} \mathrm{O}$ stratigraphy, Hole 806B. The Monterey carbon isotope excursion is clearly expressed. Note also the steepening of the thermocline indicated by both $\delta^{13} \mathrm{C}$ and $\delta^{18} \mathrm{O}$ data near $550 \mathrm{mbsf}$. The benthic $\delta^{18} \mathrm{O}$ signal begins to decline after about $510 \mathrm{mbsf}$. The "Zone N11 subevent" is clearly shown in all $\delta^{13} \mathrm{C}$ water mass monitors and is well expressed in both the deeper dwelling planktonic and benthic $\delta^{18} \mathrm{O}$ record. Solid circles = Dentoglobigerina altispira, open circles = Globoquadrina venezuelana, and squares $=$ benthic data.

increase and temperature decrease to the middle Miocene ${ }^{18} \mathrm{O}$ enrichment. As Figure 6 shows, over the interval between $15.3 \mathrm{Ma}$ (our estimate of the onset of the middle Miocene ${ }^{18} \mathrm{O}$ enrichment) and the top of the measured section at $12.4 \mathrm{Ma}$, mean benthic $\delta^{18} \mathrm{O}$ data increase by $0.8 \%$ whereas surface planktonic foraminiferal data increase by $0.4 \%$. Miller et al. (in press) have compared data from Holes 563 (North Atlantic) and 707 (western equatorial Indian Ocean) and also noted that the planktonic $\delta^{18} \mathrm{O}$ change is smaller than the benthic $\delta^{18} \mathrm{O}$ change. Their estimates are that benthic $\delta^{18} \mathrm{O}$ increased by $0.7 \%$ and planktonic $\delta^{18} \mathrm{O}$ increased by $0.4 \%$ over the middle Miocene ${ }^{18} \mathrm{O}$ "shift." Their estimates, therefore, are similar to ours, although ours are made from the same site in a western equatorial location and thus avoid problems of comparing distant records.

Miller et al. (in press) point out that this well-known difference in amplitude has been attributed either (1) to warming of equatorial surface waters during the growth of the Antarctic Ice Sheet (Shackleton and Kennett, 1975; Savin et al., 1975, 1985) or (2) to cooling of deep waters (Matthews and Poore, 1980; Prentice and Matthews, 1988 ) in addition to the ${ }^{18} \mathrm{O}$ enrichment from the growth of the Antarctic Ice Sheet.

Miller et al. (in press) suggest that the surface-water warming hypothesis is unreasonable because of planktonic and benthic foraminiferal covariance subsequent to $12.5 \mathrm{Ma}$ that implies larger ice volumes on Antarctica than the scarcity of glaciomarine sediments supports (Miller et al., 1991). They therefore conclude that the change in benthic foraminiferal values is attributable to a combination of ice-volume increase and a cooling of deep waters by $1^{\circ}-4^{\circ} \mathrm{C}$. Our data from Hole $806 \mathrm{~B}$ do not contradict this suggestion.

\section{ACKNOWLEDGMENTS}

RMC warmly thanks shipboard colleagues for their support and friendship during our 63-day sojourn on board the JOIDES Resolution during Leg 130. In particular, thanks go to Dave Mosher, Mike Prentice, Mark Leckie, Carina Lange, Johanna Resig, and Mike Storey for psychic sustenance. Our thanks to Norman Charnley for writing the stacker software used for the generation of Figures 5 and 6.

\section{REFERENCES*}

Barrera, E.B., Huber, B.T., Savin, S.M., and Webb, P.-N., 1987. Antarctic marine temperatures: late Campanian through early Paleocene. Paleoceanography, 2:21-47.

Barrera, E.B., Keller, G., and Savin, S.M., 1985. Evolution of the Miocene Ocean in the eastern North Pacific as inferred from oxygen and carbon isotopic ratios of foraminifera. In Kennett, J.P. (Ed.), The Miocene Ocean: Paleoceanography and Biogeography. Mem. Geol. Soc. Am., 163:83-102.

Berger, W.H., and Vincent, E., 1986. Deep-sea carbonates: reading the carbon isotope signal. Geol. Rundsch., 75:249-269.

Berggren, W.A., Kent, D.V., and Van Couvering, J.A., 1985. The Neogene: Part 2. Neogene geochronology and chronostratigraphy. In Snelling, N.J. (Ed.), The Chronology of the Geological Record. Geol. Soc. London Mem., 10:211-260.

Corfield, R.M., and Cartlidge, J.E., 1992. Oceanographic and climatic implications of the Palaeocene carbon isotope maximum. Terra Nova, 4:443-455.

* Abbreviations for names of organizations and publication titles in ODP reference lists follow the style given in Chemical Abstracts Service Source Index (published by American Chemical Society). 
Elderfield, H., Gieskes, J.M., Baker, P.A., Oldfield, R.K., Hawkesworth, C.J., and Miller, R., $1982 .{ }^{87} \mathrm{Sr} /{ }^{86} \mathrm{Sr}$ and ${ }^{18} \mathrm{O} /{ }^{16} \mathrm{O}$ ratios, interstitial water chemistry and diagenesis in deep-sea carbonate sediments of the Ontong-Java Plateau. Geochim. Cosmochim. Acta, 46:2259-2268.

Fairbanks, R.G., and Matthews, R.K., 1978. The marine oxygen isotopic record in Pleistocene coral, Barbados, West Indies. Quat. Res., 10:181-196.

Loutit, T.S., Pisias, N.G., and Kennett, J.P., 1983. Pacific Miocene carbon isotope stratigraphy using benthic foraminifera. Earth Planet. Sci. Lett., $66: 48-62$

Matthews, R.K., and Poore, R.Z., 1980. Tertiary ${ }^{18} \mathrm{O}$ record and glacio-eustatic sea-level fluctuations. Geology, 8:501-504.

Miller, K.G., and Curry, W.B., 1982. Eocene to Oligocene benthic foraminiferal isotope record in the Bay of Biscay. Nature, 296:347-350.

Miller, K.G., Wright, J.D., and Brower, A.N., 1989. Oligocene to Miocene stable isotope stratigraphy and planktonic foraminifer biostratigraphy of the Sierra Leone Rise (DSDP Site 366 and ODP Site 667). In Ruddiman, W., Sarnthein, M., et al., Proc. ODP, Sci. Results, 108, Pt. 2: College Station, TX (Ocean Drilling Program), 279-294.

Miller, K.G., Wright, J.D., and Fairbanks, R.G., 1991. Unlocking the IceHouse: Oligocene-Miocene oxygen isotopes, eustasy, and margin erosion. J. Geophys. Res., 96:6829-6848.

Miller, K.G., Wright, J.D., Peterson, L.C., Swart, P.K., in press. Early to middle Miocene $\delta^{18} \mathrm{O}$ : global sea level or temperature? Nature.

Prentice, M.L., and Matthews, R.K., 1988. Cenozoic ice volume history: development of a composite oxygen isotope record. Geology, 17:963-966.

Ravelo, C.A., Fairbanks, R.G., and Philander, R., 1990. Reconstructing tropical hydrography using planktonic foraminifera and an ocean model. Paleoceanography, 5:409-431.

Savin, S.M., Abel, L., Barrera, E., Hodell, D., Keller, G., Kennett, J.P., Killingley, J., Murphy, M., and Vincent, E., 1985. The evolution of Miocene surface and near-surface marine temperatures: oxygen isotopic evidence. In Kennett, J.P. (Ed.), The Miocene Ocean: Paleoceanography and Biogeography. Mem. Geol. Soc. Am., 163:49-82.

Savin, S.M., Douglas, R.G., and Stehli, F.G., 1975. Tertiary marine paleotemperatures. Geol. Soc. Am. Bull., 86:1499-1510.

Savin, S.M., Keller, G., Douglas, R.G., Killingley, J.S., Shaughnessy, L., Sommer, M.A., Vincent, E., and Woodruff, F., 1981. Miocene benthic foraminiferal isotope records: a synthesis. Mar. Micropaleontol., 6:423-450.

Shackleton, N.J., 1967. Oxygen isotope analyses and Pleistocene temperatures re-assessed. Nature, 215:15-17.

, 1982. The deep-sea sediment record of climate variability. Progr. Oceanogr., 11:199-218.

Shackleton, N.J., Hall, M.A., and Bleil, U., 1985. Carbon isotope stratigraphy, Site 577. In Heath, G.R., Burckle, L.H., et al., Init. Repts. DSDP, 86: Washington (U.S. Govt. Printing Office), 503-512.
Shackleton, N.J., Hall, M.A., and Boersma, A., 1984. Oxygen and carbon isotope data from Leg 74 foraminifers. In Moore, T.C., Jr., Rabinowitz, P.D., et al., Init. Repts. DSDP, 74: Washington (U.S. Govt. Printing Office), 599-612.

Shackleton, N.J., and Kennett, J.P., 1975. Paleotemperature history of the Cenozoic and the initiation of Antarctic glaciation: oxygen and carbon isotope analyses in DSDP Sites 277, 279, and 281. In Kennett, J.P., Houtz, R.E., et al., Init. Repts. DSDP, 29: Washington (U.S. Govt. Printing Office), 743-755.

Shackleton, N.J., and Opdyke, N.D., 1973. Oxygen isotope and paleomagnetic stratigraphy of equatorial Pacific Core V28-238: oxygen isotope temperatures and ice volumes on a $10^{5}$ year and $10^{6}$ year scale. Quat. Res., 3:39-55. ,- 1976 . Oxygen isotope and paleomagnetic stratigraphy of Pacific Core V28-239, late Pliocene to Pleistocene. Geol. Soc. Am. Bull., 145:449-464.

Vincent, E., and Berger, W.H., 1985. Carbon dioxide and polar cooling in the Miocene: the Monterey hypothesis. In Sundquist, E.T., and Broecker, W.S. (Eds.), The Carbon Cycle and Atmospheric $\mathrm{CO}_{2}:$ Natural Variations Archean to Present. Am. Geophys. Union Monogr., 32:455-468.

Vincent, E., Killingley, J.S., and Berger, W.H., 1985. Miocene oxygen and carbon isotope stratigraphy of the tropical Indian Ocean. In Kennett, J.P. (Ed.) The Miocene Ocean: Paleoceanography and Biogeography. Mem. Geol. Soc. Am., 163:103-130.

Woodruff, F., and Savin, S.M., 1989. Miocene deep-water oceanography. Paleoceanography, 4:87-140.

Woodruff, F., Savin, S.M., and Douglas, R.G., 1981. Miocene stable isotope record: a detailed deep Pacific ocean study and its paleoclimatic implications. Science, 212:665-668.

Wright, J.D., and Miller, K.G., 1992. Miocene stable isotope stratigraphy, Site 747, Kerguelen Plateau. In Wise, S.W., Jr., Schlich, R., et al., Proc. ODP, Sci. Results, 120, Pt. 2: College Station, TX (Ocean Drilling Program), 855-866.

Wright, J.D., Miller, K.G., and Fairbanks, R.G., 1991. Evolution of modern deep-water circulation: evidence from the late Miocene southern ocean. Paleoceanography, 6:275-290.

, 1992. Early and middle Miocene stable isotopes: implications for deep-water circulation and climate. Paleoceanography, 7:357-389.

Date of initial receipt: 30 August 1991

Date of acceptance: 3 September 1992

Ms 130B-026 

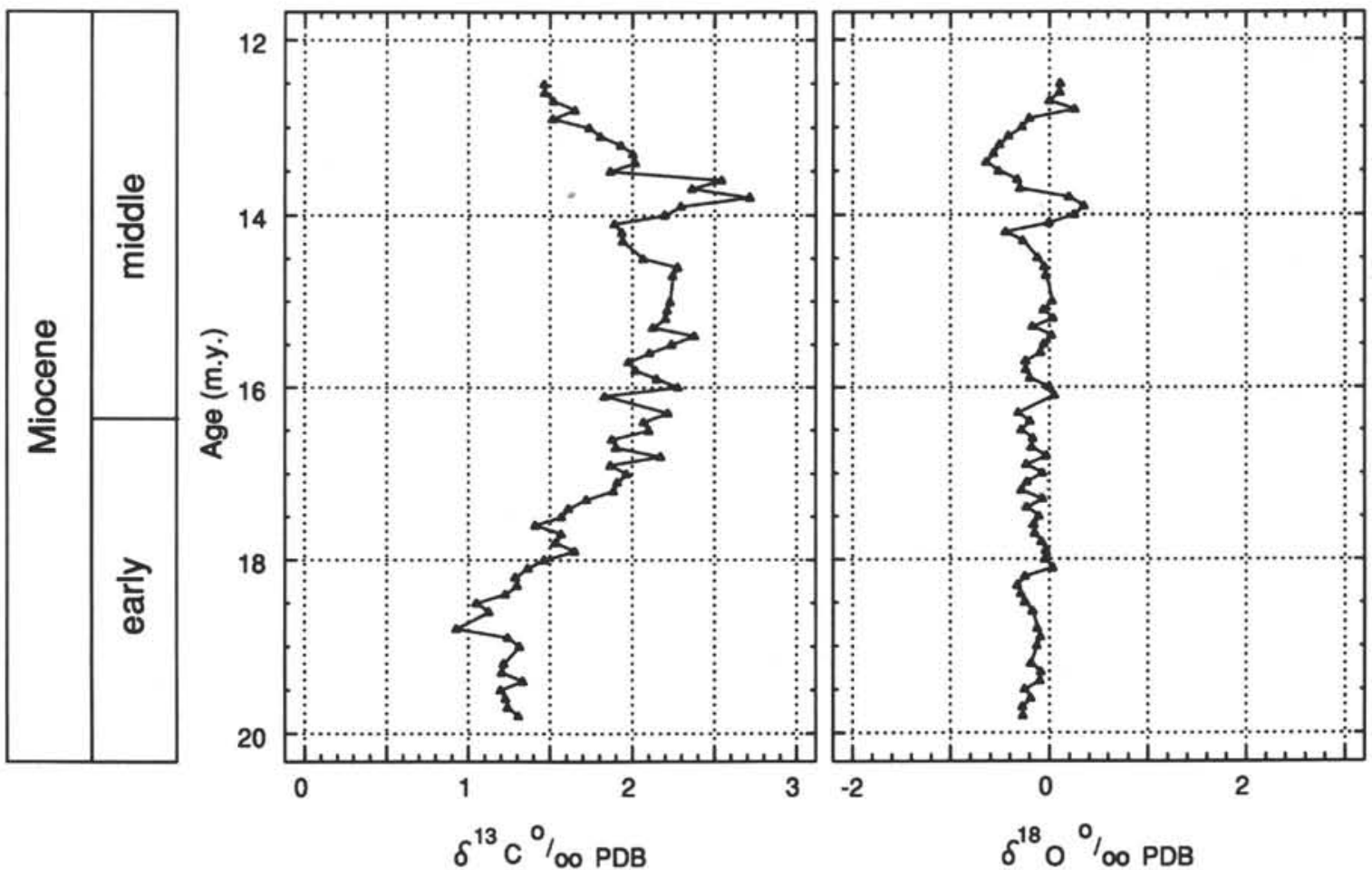

Figure 5. Chronostratigraphy of bulk sample $\delta^{13} \mathrm{C}$ and $\delta^{18} \mathrm{O}$ measurements, Hole 806B. These $\delta^{13} \mathrm{C}$ data show the onset of the middle Miocene Monterey excursion at $18.8 \mathrm{Ma}$ and its decline following the "Zone N11 subevent" at $14.5 \mathrm{Ma}$. The $\delta^{18} \mathrm{O}$ data are more monotonous except for the increase in $\delta^{18} \mathrm{O}$ values during the "Zone N11 subevent."

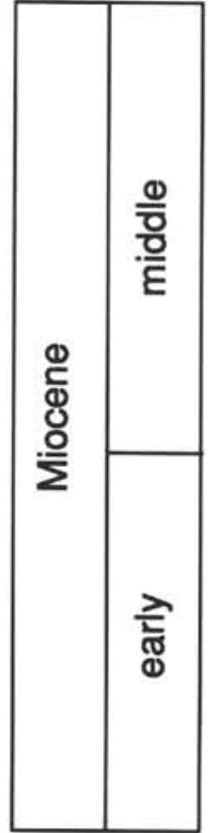

- D. altispira

- Gq. venezuelana

- Benthics
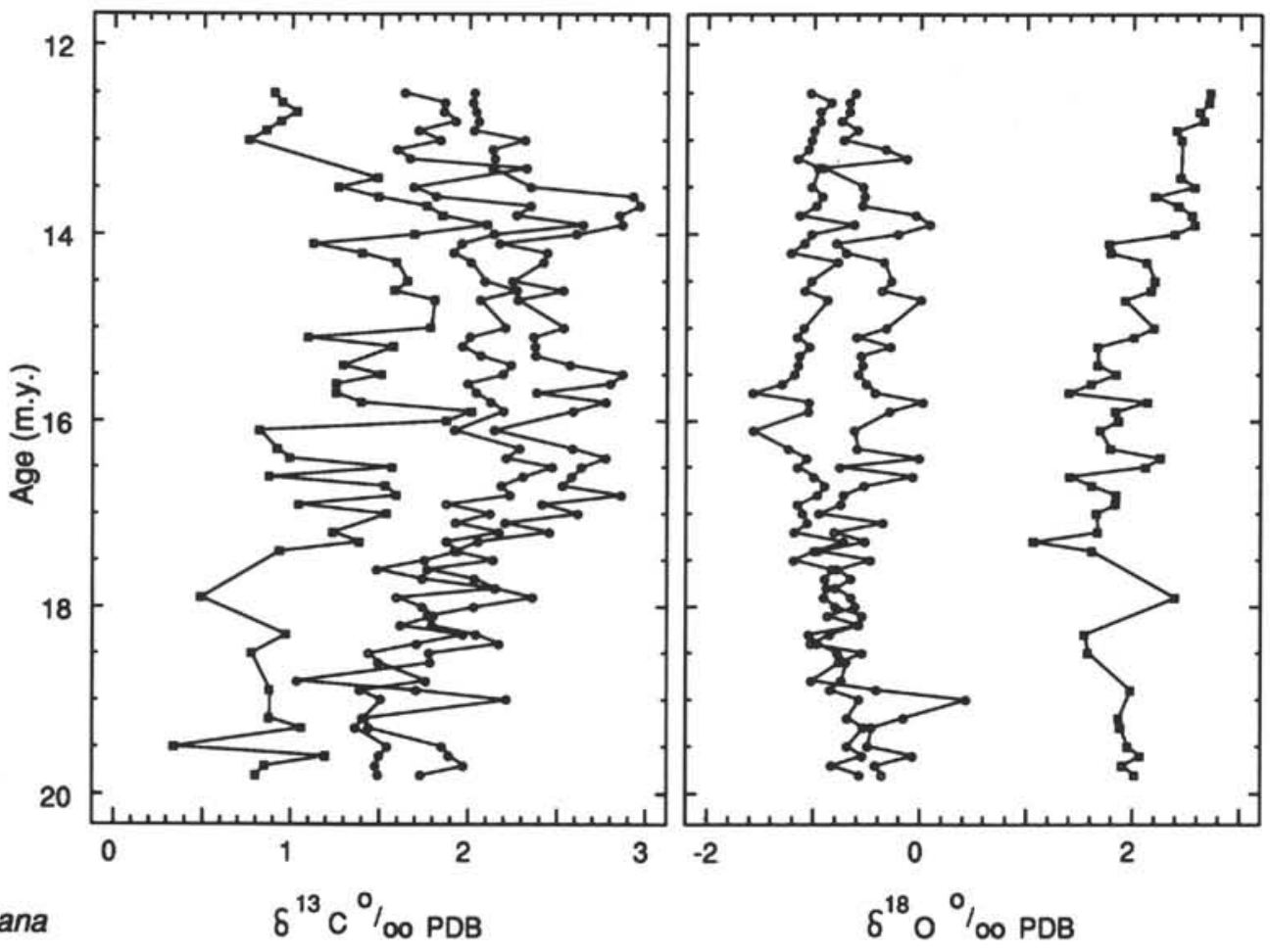

Figure 6. Chronostratigraphy of foraminiferal $\delta^{13} \mathrm{C}$ and $\delta^{18} \mathrm{O}$ measurements, Hole 806B. The chronology of events is discussed in the text. Note the increase in $\delta^{18} \mathrm{O}$ values starting at $15.3 \mathrm{Ma}$. With the exception of the data set discussed by Shackleton et al. (1984), our estimate of the onset of the $\delta^{18} \mathrm{O}$ increase is 1-2 m.y. older than other published estimates (e.g., Barrera et al., 1985), although our estimates are provisional (as discussed in the text) because they are based solely on nannofossil age control points. Symbols as for Figure 4 . 


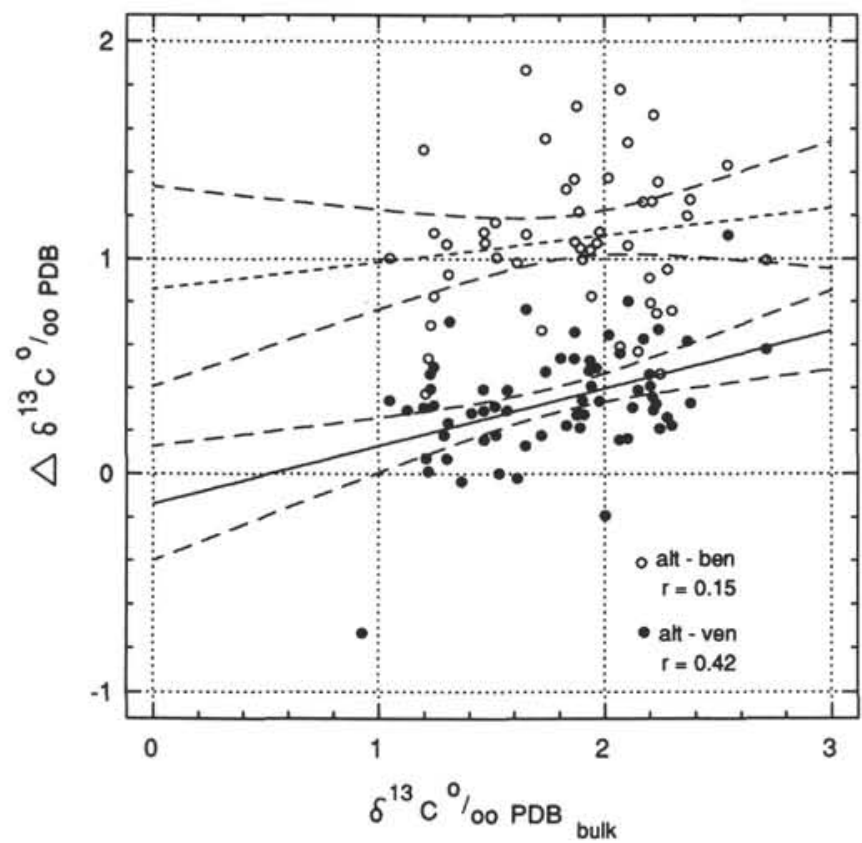

Figure 7. Two estimates of the vertical carbon isotope gradient in the middle Miocene Pacific Ocean. The increase in $\Delta \delta^{13} \mathrm{C}_{\text {alt-ven }}$ suggests a minor contribution from surface-water productivity to the structure of the Monterey carbon isotope excursion. However, $\Delta \delta^{13} \mathrm{C}_{\text {alt-ben }}$ are not even weakly correlated, suggesting that the major component of the Monterey carbon isotope excursion is caused by between-reservoir fractionation of carbon isotopes. 
APPENDIX A

Bulk-rock $\delta^{13} \mathrm{C}$ and $\delta^{18} \mathrm{O}$ Data from Hole 805B

\begin{tabular}{|c|c|c|c|c|c|c|c|c|c|c|c|c|c|}
\hline $\begin{array}{l}\text { Depth } \\
\text { (mbsf) }\end{array}$ & $\begin{array}{l}\text { Age } \\
\text { (Ma) }\end{array}$ & Lab no. & $\begin{array}{l}\text { Core, section, } \\
\text { interval }(\mathrm{cm})\end{array}$ & Species & $\delta^{13} \mathrm{C}$ & $\delta^{18} \mathrm{O}$ & $\begin{array}{l}\text { Depth } \\
\text { (mbsf) }\end{array}$ & $\begin{array}{l}\text { Age } \\
(\mathrm{Ma})\end{array}$ & Lab no. & $\begin{array}{l}\text { Core, section, } \\
\text { interval }(\mathrm{cm})\end{array}$ & Species & $\delta^{13} \mathrm{C}$ & $\delta^{18} \mathrm{O}$ \\
\hline 292.39 & 11.53 & A90/4012 & $32 \mathrm{R}-1,19$ & Bulk & 1.62 & -0.08 & 311.99 & 12.90 & A90/4161 & $34 \mathrm{R}-1,99$ & Bulk & 2.22 & -0.03 \\
\hline 292.59 & 11.55 & A $90 / 4013$ & $32 \mathrm{R}-1,39$ & Bulk & 1.53 & -0.19 & 312.19 & 12.91 & A90/4162 & $34 \mathrm{R}-1,119$ & Bulk & 2.22 & -0.03 \\
\hline 292.79 & 11.56 & A90/3717 & $32 \mathrm{R}-1,59$ & Bulk & 1.32 & -0.11 & 312.39 & 12.92 & A90/4163 & $34 \mathrm{R}-1,139$ & Bulk & 2.29 & -0.04 \\
\hline 292.99 & 11.58 & A90/4014 & $32 \mathrm{R}-1,79$ & Bulk & 1.32 & 0.02 & 312.59 & 12.93 & A90/4164 & $34 \mathrm{R}-2,9$ & Bulk & 2.28 & 0.16 \\
\hline 293.19 & 11.59 & A90/4015 & $32 \mathrm{R}-1,99$ & Bulk & 1.35 & 0.17 & 312.79 & 12.94 & A90/4565 & $34 \mathrm{R}-2,29$ & Bulk & 2.10 & -0.03 \\
\hline 293.39 & 11.60 & A90/4016 & $32 \mathrm{R}-1,119$ & Bulk & 1.27 & -0.05 & 312.99 & 12.94 & A90/4566 & $34 \mathrm{R}-2,49$ & Bulk & 2.01 & 0.12 \\
\hline 293.59 & 11.62 & A $90 / 4017$ & $32 \mathrm{R}-1,139$ & Bulk & 1.47 & -0.07 & 313.19 & 12.95 & A90/3729 & $34 \mathrm{R}-2,69$ & Bulk & 1.87 & 0.05 \\
\hline 293.79 & 11.63 & A90/4018 & $32 \mathrm{R}-2,9$ & Bulk & 1.44 & -0.05 & 313.39 & 12.96 & A90/4567 & $34 \mathrm{R}-2,89$ & Bulk & 1.93 & 0.11 \\
\hline 293.99 & 11.65 & A90/4019 & $32 \mathrm{R}-2,29$ & Bulk & 1.49 & -0.12 & 313.59 & 12.97 & A90/4568 & $34 \mathrm{R}-2,109$ & Bulk & 2.01 & -0.26 \\
\hline 294.19 & 11.66 & A90/4020 & $32 \mathrm{R}-2,49$ & Bulk & 1.68 & 0.01 & 313.79 & 12.98 & A90/4569 & $34 \mathrm{R}-2,129$ & Bulk & 1.91 & -0.08 \\
\hline 294.39 & 11.68 & A90/3718 & $32 \mathrm{R}-2,69$ & Bulk & 1.40 & -0.05 & 313.99 & 12.99 & A90/4570 & $34 \mathrm{R}-2,149$ & Bulk & 1.97 & -0.26 \\
\hline 294.57 & 11.69 & A $90 / 4021$ & $32 \mathrm{R}-2,87$ & Bulk & 1.28 & -0.30 & 314.19 & 13.00 & A90/4571 & $34 \mathrm{R}-3,19$ & Bulk & 2.11 & -0.13 \\
\hline 294.79 & 11.71 & A90/4022 & $32 \mathrm{R}-2,109$ & Bulk & 1.48 & -0.29 & 314.39 & 13.01 & A90/4572 & $34 \mathrm{R}-3,39$ & Bulk & 2.01 & -0.03 \\
\hline 295.39 & 11.75 & A90/4023 & $32 \mathrm{R}-3,19$ & Bulk & 1.46 & 0.35 & 314.59 & 13.02 & A $90 / 3730$ & $34 \mathrm{R}-3,59$ & Bulk & 1.93 & 0.22 \\
\hline 295.59 & 11.77 & A90/4024 & $32 \mathrm{R}-3,39$ & Bulk & 1.62 & 0.11 & 314.79 & 13.02 & A90/4573 & $34 \mathrm{R}-3,79$ & Bulk & 1.89 & -0.05 \\
\hline 295.79 & 11.78 & A90/3719 & $32 \mathrm{R}-3,59$ & Bulk & 1.50 & 0.19 & 314.99 & 13.03 & A $90 / 4574$ & $34 \mathrm{R}-2,99$ & Bulk & 2.01 & -0.03 \\
\hline 295.99 & 11.79 & A90/4025 & $32 \mathrm{R}-3,79$ & Bulk & 1.75 & 0.16 & 315.19 & 13.04 & A90/4575 & $34 \mathrm{R}-3,119$ & Bulk & 2.02 & -0.13 \\
\hline 296.19 & 11.81 & A90/4026 & $32 \mathrm{R}-3,99$ & Bulk & 1.55 & 0.14 & 315.39 & 13.05 & A $90 / 4576$ & $34 \mathrm{R}-3,139$ & Bulk & 2.08 & -0.01 \\
\hline 296.39 & 11.82 & A $90 / 4027$ & $32 \mathrm{R}-3,119$ & Bulk & 1.59 & -0.04 & 315.59 & 13.06 & A90/4577 & $34 \mathrm{R}-4,9$ & Bulk & 2.07 & -0.05 \\
\hline 296.59 & 11.84 & A90/4028 & $32 \mathrm{R}-3,139$ & Bulk & 1.60 & 0.11 & 315.79 & 13.07 & A90/4578 & $34 \mathrm{R}-4,29$ & Bulk & 2.05 & -0.13 \\
\hline 296.79 & 11.85 & A90/4029 & $32 R-4,9$ & Bulk & 1.74 & -0.05 & 315.99 & 13.08 & A $90 / 4579$ & $34 \mathrm{R}-4,49$ & Bulk & 2.05 & -0.18 \\
\hline 296.99 & 11.87 & A $90 / 4030$ & $32 R-4,29$ & Bulk & 1.92 & 0.13 & 316.19 & 13.09 & A $90 / 3731$ & $34 R-4,69$ & Bulk & 2.13 & -0.41 \\
\hline 297.19 & 11.88 & A90/4031 & $32 \mathrm{R}-4,49$ & Bulk & 1.69 & 0.10 & 316.39 & 13.10 & A90/4580 & $34 \mathrm{R}-4,89$ & Bulk & 2.06 & -0.09 \\
\hline 297.39 & 11.90 & A $90 / 3720$ & $32 \mathrm{R}-4,69$ & Bulk & 1.65 & 0.21 & 316.59 & 13.11 & A90/4581 & $34 \mathrm{R}-4,109$ & Bulk & 1.94 & -0.07 \\
\hline 297.59 & 11.91 & A90/4032 & $32 \mathrm{R}-4,89$ & Bulk & 1.81 & 0.21 & 316.79 & 13.12 & A90/4582 & $34 \mathrm{R}-4,129$ & Bulk & 2.11 & -0.28 \\
\hline 297.79 & 11.93 & A $90 / 4033$ & $32 R-4,109$ & Bulk & 1.87 & 0.18 & 316.99 & 13.13 & A90/4583 & $34 \mathrm{R}-4,149$ & Bulk & 2.10 & -0.28 \\
\hline 297.99 & 11.94 & A $90 / 4034$ & $32 \mathrm{R}-4,129$ & Bulk & 1.34 & -0.04 & 317.19 & 13.14 & A $90 / 4584$ & $34 \mathrm{R}-5,19$ & Bulk & 2.19 & -0.15 \\
\hline 298.18 & 11.95 & A90/4035 & $32 R-4,148$ & Bulk & 1.59 & 0.15 & 317.39 & 13.15 & A90/4585 & $34 \mathrm{R}-5,39$ & Bulk & 2.13 & -0.16 \\
\hline 298.39 & 11.97 & A90/4036 & $32 \mathrm{R}-5,19$ & Bulk & 1.77 & 0.24 & 317.59 & 13.16 & A90/3732 & $34 \mathrm{R}-5,59$ & Bulk & 1.98 & -0.14 \\
\hline 298.59 & 11.98 & A90/4037 & $32 \mathrm{R}-5,39$ & Bulk & 1.94 & 0.06 & 317.79 & 13.17 & A $90 / 4586$ & $34 \mathrm{R}-5,79$ & Bulk & 2.21 & 0.00 \\
\hline 298.79 & 12.00 & A90/3721 & $32 R-5,59$ & Bulk & 1.71 & 0.17 & 317.99 & 13.18 & A90/4587 & $34 \mathrm{R}-5,99$ & Bulk & 2.23 & -0.12 \\
\hline 298.99 & 12.01 & A90/4038 & $32 \mathrm{R}-5,79$ & Bulk & 1.60 & 0.20 & 318.19 & 13.19 & A90/4588 & $34 \mathrm{R}-5,119$ & Bulk & 2.12 & -0.02 \\
\hline 301.59 & 12.20 & A90/4039 & $33 \mathrm{R}-1,19$ & Bulk & 1.58 & 0.29 & 318.39 & 13.20 & A90/4589 & $34 \mathrm{R}-5,139$ & Bulk & 2.28 & 0.12 \\
\hline 301.79 & 12.22 & A $90 / 4040$ & $33 R-1,39$ & Bulk & 1.64 & 0.23 & 320.69 & 13.32 & A $90 / 4590$ & $35 \mathrm{R}-1,19$ & Bulk & 2.28 & -0.11 \\
\hline 301.99 & 12.23 & A $90 / 3722$ & $33 \mathrm{R}-1,59$ & Bulk & 1.54 & 0.02 & 320.69 & 13.32 & A90/4639 & $35 \mathrm{R}-1,19$ & Bulk & 2.15 & -0.34 \\
\hline 302.19 & 12.25 & A $90 / 4041$ & $33 \mathrm{R}-1,79$ & Bulk & 1.53 & 0.36 & 320.89 & 13.33 & A $90 / 4640$ & $35 \mathrm{R}-1,39$ & Bulk & 2.13 & -0.17 \\
\hline 302.39 & 12.26 & A $90 / 4042$ & $33 \mathrm{R}-1,99$ & Bulk & 1.75 & 0.28 & 321.09 & 13.34 & A $90 / 3733$ & $35 \mathrm{R}-1,59$ & Bulk & 2.13 & -0.02 \\
\hline 302.59 & 12.28 & A90/4043 & $33 \mathrm{R}-1,119$ & Bulk & 1.76 & 0.02 & 321.29 & 13.35 & A90/4641 & $35 \mathrm{R}-1,79$ & Bulk & 2.11 & -0.03 \\
\hline 302.99 & 12.31 & A90/4115 & $33 R-2,9$ & Bulk & 1.65 & 0.09 & 321.49 & 13.36 & A $90 / 4642$ & $35 \mathrm{R}-1,99$ & Bulk & 2.28 & 0.15 \\
\hline 303.19 & 12.32 & A90/4116 & $33 \mathrm{R}-2,29$ & Bulk & 1.57 & 0.07 & 321.69 & 13.37 & A $90 / 4643$ & $35 \mathrm{R}-1,119$ & Bulk & 2.33 & 0.08 \\
\hline 303.39 & 12.34 & A90/4117 & $33 \mathrm{R}-2,49$ & Bulk & 1.55 & 0.11 & 321.89 & 13.38 & A90/4644 & $35 \mathrm{R}-1,139$ & Bulk & 2.41 & 0.18 \\
\hline 303.59 & 12.35 & A $90 / 3723$ & $33 R-2,69$ & Bulk & 1.68 & 0.35 & 322.09 & 13.40 & A90/4645 & $35 \mathrm{R}-2,9$ & Bulk & 2.45 & 0.14 \\
\hline 303.79 & 12.36 & A90/4118 & $33 \mathrm{R}-2,89$ & Bulk & 1.64 & 0.24 & 322.29 & 13.41 & A90/4646 & $35 \mathrm{R}-2,29$ & Bulk & 2.47 & 0.17 \\
\hline 304.21 & 12.40 & A90/4119 & $33 \mathrm{R}-2,131$ & Bulk & 1.47 & 0.04 & 322.49 & 13.42 & A90/4647 & $35 \mathrm{R}-2,49$ & Bulk & 2.32 & 0.01 \\
\hline 304.38 & 12.41 & A90/4120 & $33 \mathrm{R}-2,148$ & Bulk & 1,42 & 0.32 & 322.69 & 13.43 & A90/3734 & $35 \mathrm{R}-2,69$ & Bulk & 2.29 & 0.15 \\
\hline 304.59 & 12.42 & A $90 / 4121$ & $33 \mathrm{R}-3,19$ & Bulk & 1.51 & 0.15 & 322.89 & 13.44 & A $90 / 4648$ & $35 \mathrm{R}-2,89$ & Bulk & 2.35 & 0.10 \\
\hline 304.79 & 12.44 & A90/4122 & $33 R-3,39$ & Bulk & 1.56 & 0.03 & 323,29 & 13.46 & A $90 / 4650$ & $35 \mathrm{R}-2,129$ & Bulk & 2.24 & -0.06 \\
\hline 304.99 & 12.45 & A90/3724 & $33 \mathrm{R}-3,59$ & Bulk & 1.49 & 0.12 & 323.69 & 13.48 & A $90 / 4652$ & $35 \mathrm{R}-3,19$ & Bulk & 2.27 & -0.12 \\
\hline 305.19 & 12.47 & A90/4123 & $33 \mathrm{R}-3,79$ & Bulk & 1.64 & 0.38 & 323.89 & 13.49 & A90/4653 & $35 \mathrm{R}-3,39$ & Bulk & 2.23 & 0.11 \\
\hline 305.39 & 12.48 & A90/4124 & $33 R-3,99$ & Bulk & 1.54 & 0.27 & 324.09 & 13.50 & A90/3774 & $35 \mathrm{R}-3,59$ & Bulk & 2.32 & 0.08 \\
\hline 305.79 & 12.51 & A90/4127 & $33 R-3,139$ & Bulk & 1.65 & -0.11 & 324.49 & 13.52 & A90/4655 & $35 R-3,99$ & Bulk & 2.29 & 0.06 \\
\hline 305.99 & 12.53 & A90/4128 & $33 R-4,9$ & Bulk & 1.97 & -0.31 & 325.09 & 13.55 & A90/4656 & $35 \mathrm{R}-4,9$ & Bulk & 2.57 & 0.16 \\
\hline 306.19 & 12.54 & A $90 / 4129$ & $33 R-4,29$ & Bulk & 2.08 & 0.08 & 325.69 & 13.58 & A90/3775 & $35 R-4,69$ & Bulk & 2.50 & -0.24 \\
\hline 306.39 & 12.55 & A $90 / 4130$ & $33 R-4,49$ & Bulk & 1.95 & 0.00 & 325.89 & 13.60 & A90/4659 & $35 \mathrm{R}-4,89$ & Bulk & 2.65 & 0.04 \\
\hline 306.59 & 12.57 & A90/3725 & $33 R-4,69$ & Bulk & 2.29 & 0.51 & 326.09 & 13.61 & A $90 / 4660$ & $35 \mathrm{R}-4,109$ & Bulk & 2.68 & -0.03 \\
\hline 306.79 & 12.58 & A $90 / 4131$ & $33 \mathrm{R}-4,89$ & Bulk & 2.01 & 0.06 & 326.29 & 13.63 & A90/4661 & $35 \mathrm{R}-4,129$ & Bulk & 2.54 & -0.07 \\
\hline 306.99 & 12.60 & A90/4132 & $33 R-4,109$ & Bulk & 2.01 & -0.01 & 327.09 & 13.69 & A90/3776 & $35 R-5,59$ & Bulk & 2.56 & -0.10 \\
\hline 307.22 & 12.62 & A90/4133 & $33 \mathrm{R}-4,132$ & Bulk & 1.93 & -0.05 & 330.19 & 13.94 & A91/3221 & $36 \mathrm{R}-1,19$ & Bulk & 2.30 & 0.33 \\
\hline 307.38 & 12.63 & A90/4134 & $33 \mathrm{R}-4,148$ & Bulk & 1.96 & -0.16 & 330.39 & 13.96 & A91/3222 & $36 \mathrm{R}-1,39$ & Bulk & 2.24 & 0.37 \\
\hline 307.59 & 12.64 & A90/4135 & $33 \mathrm{R}-5,19$ & Bulk & 1.93 & 0.06 & 330.59 & 13.98 & A90/3777 & $36 \mathrm{R}-1,59$ & Bulk & 2.15 & -0.05 \\
\hline 307.79 & 12.66 & A90/4136 & $33 \mathrm{R}-5,39$ & Bulk & 2.10 & 0.00 & 330.79 & 13.99 & A $91 / 3223$ & $36 \mathrm{R}-1,79$ & Bulk & 2.18 & 0.24 \\
\hline 307.99 & 12.67 & A90/3726 & $33 R-5,59$ & Bulk & 2.48 & 0.14 & 330.99 & 14.01 & A $91 / 3224$ & $36 \mathrm{R}-1,99$ & Bulk & 2.03 & 0.14 \\
\hline 308.19 & 12.69 & A90/4137 & $33 R-5,79$ & Bulk & 2.10 & 0.08 & 331.19 & 14.03 & A91/3225 & $36 \mathrm{R}-1,119$ & Bulk & 1.99 & 0.07 \\
\hline 308.39 & 12.70 & A90/4138 & $33 \mathrm{R}-5,99$ & Bulk & 1.93 & -0.02 & 331.39 & 14.04 & A91/3226 & $36 \mathrm{R}-1,139$ & Bulk & 2.04 & 0.12 \\
\hline 308.59 & 12.72 & A90/4139 & $33 \mathrm{R}-5,119$ & Bulk & 2.39 & 0.16 & 331.59 & 14.06 & A91/3227 & $36 \mathrm{R}-2,9$ & Bulk & 1.97 & 0.23 \\
\hline 308.79 & 12.73 & A $90 / 4140$ & $33 R-5,139$ & Bulk & 2.20 & 0.09 & 331.79 & 14.08 & A91/3228 & $36 \mathrm{R}-2,29$ & Bulk & 1.80 & 0.22 \\
\hline 308.99 & 12.74 & A90/4153 & $33 R-6,9$ & Bulk & 1.97 & 0.13 & 331.99 & 14.09 & A91/3229 & $36 \mathrm{R}-2,49$ & Bulk & 2.23 & 0.09 \\
\hline 309.19 & 12.76 & A90/4154 & $33 \mathrm{R}-6,29$ & Bulk & 2.15 & 0.28 & 332.19 & 14.11 & A90/3778 & $36 \mathrm{R}-2,69$ & Bulk & 2.08 & 0.02 \\
\hline 309.40 & 12.77 & A90/4155 & $33 R-6,50$ & Bulk & 2.02 & 0.30 & 332.39 & 14.13 & A $91 / 3230$ & $36 \mathrm{R}-2,89$ & Bulk & 2.14 & 0.36 \\
\hline 309.59 & 12.79 & A90/3727 & $33 R-6,69$ & Bulk & 2.01 & -0.07 & 332.59 & 14.14 & A $91 / 3231$ & $36 \mathrm{R}-2,109$ & Bulk & 2.19 & 0.23 \\
\hline 309.79 & 12.80 & A90/4156 & $33 R-6,89$ & Bulk & 2.12 & 0.00 & 332.79 & 14.16 & A91/3286 & $36 \mathrm{R}-2,129$ & Bulk & 2.08 & 0.43 \\
\hline 309.99 & 12.81 & A90/4157 & $33 R-6,109$ & Bulk & 2.25 & -0.05 & 332.98 & 14.17 & A91/3287 & $36 \mathrm{R}-2,148$ & Bulk & 2.07 & 0.13 \\
\hline 311.19 & 12.86 & A90/4158 & $34 \mathrm{R}-1,19$ & Bulk & 2.14 & -0.12 & 333.19 & 14.19 & A91/3288 & $36 \mathrm{R}-3,19$ & Bulk & 2.08 & 0.16 \\
\hline 311.59 & 12.88 & A90/3728 & $34 \mathrm{R}-1,59$ & Bulk & 1.98 & 0.02 & 333.39 & 14.21 & A91/3289 & $36 \mathrm{R}-3,39$ & Bulk & 2.10 & 0.36 \\
\hline
\end{tabular}


Appendix A (continued).

\begin{tabular}{|c|c|c|c|c|c|c|c|c|c|c|c|c|c|}
\hline $\begin{array}{l}\text { Depth } \\
\text { (mbsf) }\end{array}$ & $\begin{array}{l}\text { Age } \\
\text { (Ma) }\end{array}$ & Lab no. & $\begin{array}{l}\text { Core, section, } \\
\text { interval }(\mathrm{cm})\end{array}$ & Species & $\delta^{13} \mathrm{C}$ & $\delta^{18} \mathrm{O}$ & $\begin{array}{l}\text { Depth } \\
\text { (mbsf) }\end{array}$ & $\begin{array}{l}\text { Age } \\
(\mathrm{Ma})\end{array}$ & Lab no. & $\begin{array}{l}\text { Core, section, } \\
\text { interval }(\mathrm{cm})\end{array}$ & Species & $\delta^{13} \mathrm{C}$ & $\delta^{18} \mathrm{O}$ \\
\hline 333.59 & 14.22 & A90/3779 & $36 \mathrm{R}-3,59$ & Bulk & 2.06 & -0.06 & 352.79 & 15.80 & A91/3359 & $38 \mathrm{R}-3,39$ & Bulk & 2.53 & 0.17 \\
\hline 333.79 & 14.24 & A91/3290 & $36 \mathrm{R}-3,79$ & Bulk & 2.10 & 0.03 & 352.99 & 15.81 & A90/3791 & $38 \mathrm{R}-3,59$ & Bulk & 2.49 & 0.40 \\
\hline 333.99 & 14.26 & A91/3291 & $36 \mathrm{R}-3,99$ & Bulk & 2.29 & 0.00 & 353.19 & 15.83 & A91/3360 & $38 \mathrm{R}-3,79$ & Bulk & 2.67 & 0.23 \\
\hline 334.19 & 14.27 & A91/3292 & $36 \mathrm{R}-3,119$ & Bulk & 2.12 & -0.02 & 353.39 & 15.85 & A $91 / 3361$ & $38 \mathrm{R}-3,99$ & Bulk & 2.63 & 0.17 \\
\hline 334.39 & 14.29 & A91/3293 & $36 \mathrm{R}-3,139$ & Bulk & 1.94 & -0.03 & 353.99 & 15.90 & A91/3362 & $38 \mathrm{R}-4,9$ & Bulk & 2.34 & 0.18 \\
\hline 334.59 & 14.31 & A91/3294 & $36 \mathrm{R}-4,9$ & Bulk & 2.11 & 0.04 & 354.19 & 15.91 & A91/3363 & $38 \mathrm{R}-4,29$ & Bulk & 2.39 & 0.13 \\
\hline 334.79 & 14.32 & A91/3295 & $36 \mathrm{R}-4,29$ & Bulk & 2.12 & 0.03 & 354.39 & 15.93 & A91/3364 & $38 \mathrm{R}-4,49$ & Bulk & 2.37 & 0.06 \\
\hline 334.99 & 14.34 & A91/3296 & $36 \mathrm{R}-4,49$ & Bulk & 2.00 & 0.14 & 354.59 & 15.94 & A90/3792 & $38 R-4,69$ & Bulk & 2.40 & 0.24 \\
\hline 335.19 & 14.35 & A $90 / 3780$ & $36 \mathrm{R}-4,69$ & Bulk & 2.05 & -0.09 & 354.79 & 15.96 & A91/3365 & $38 \mathrm{R}-4,89$ & Bulk & 2.40 & 0.12 \\
\hline 335.39 & 14.37 & A91/3297 & $36 \mathrm{R}-4,89$ & Bulk & 2.25 & 0.04 & 354.99 & 15.98 & A91/3366 & $38 \mathrm{R}-4,109$ & Bulk & 2.56 & 0.19 \\
\hline 335.59 & 14.39 & A91/3298 & $36 \mathrm{R}-4,109$ & Bulk & 2.03 & 0.10 & 355.19 & 15.99 & A91/3367 & $38 \mathrm{R}-4,129$ & Bulk & 2.43 & 0.02 \\
\hline 335.79 & 14.40 & A91/3299 & $36 \mathrm{R}-4,129$ & Bulk & 2.12 & 0.14 & 355.38 & 16.01 & A91/3368 & $38 \mathrm{R}-4,148$ & Bulk & 2.50 & 0.01 \\
\hline 335.98 & 14.42 & A91/3300 & $36 \mathrm{R}-4,148$ & Bulk & 2.30 & 0.13 & 355.59 & 16.03 & A91/3369 & $38 \mathrm{R}-5,19$ & Bulk & 2.52 & -0.02 \\
\hline 336.19 & 14.44 & A91/3301 & $36 \mathrm{R}-5,19$ & Bulk & 2.30 & 0.28 & 355.79 & 16.04 & A $91 / 3370$ & $38 \mathrm{R}-5,39$ & Bulk & 2.86 & 0.28 \\
\hline 336.39 & 14,45 & A91/3302 & $36 \mathrm{R}-5,39$ & Bulk & 2.74 & 0.48 & 355.99 & 16.06 & A $90 / 3793$ & $38 \mathrm{R}-5,59$ & Bulk & 2.56 & 0.30 \\
\hline 36.59 & 14.47 & A90/3781 & $36 \mathrm{R}-5,59$ & Bulk & 2.22 & 0.30 & 356.19 & 16.08 & A91/3371 & $38 \mathrm{R}-5,79$ & Bulk & 2.33 & 0.32 \\
\hline 336.79 & 14.49 & A91/3303 & $36 \mathrm{R}-5,79$ & Bulk & 2.13 & 0.34 & 356.39 & 16.09 & A $91 / 3372$ & $38 \mathrm{R}-5,99$ & Bulk & 2.20 & 0.09 \\
\hline 336.99 & 14.50 & A91/3304 & $36 \mathrm{R}-5,99$ & Bulk & 2.37 & 0.33 & 356.59 & 16.11 & A $91 / 3373$ & $38 \mathrm{R}-5,119$ & Bulk & 2.42 & -0.12 \\
\hline 337.19 & 14.52 & A91/3305 & $36 \mathrm{R}-5,119$ & Bulk & 2.30 & 0.08 & 359.19 & 16.32 & A91/3374 & $39 \mathrm{R}-1,19$ & Bulk & 2.36 & 0.20 \\
\hline 337.39 & 14.53 & A91/3306 & $36 \mathrm{R}-5,139$ & Bulk & 2.21 & 0.03 & 359.39 & 16.34 & A91/3375 & $39 \mathrm{R}-1,39$ & Bulk & 2.37 & 0.23 \\
\hline 337.59 & 14.55 & A90/3782 & $36 \mathrm{R}-6,9$ & Bulk & 2.21 & -0.07 & 359.61 & 16.36 & A90/3794 & $39 \mathrm{R}-1,61$ & Bulk & 2.39 & 0.30 \\
\hline 339.89 & 14.74 & A91/3307 & $37 \mathrm{R}-1,19$ & Bulk & 2.31 & 0.07 & 359.79 & 16.37 & A91/3376 & $39 \mathrm{R}-1,79$ & Bulk & 2.27 & -0.03 \\
\hline 340.09 & 14.76 & A91/3308 & $37 \mathrm{R}-1,39$ & Bulk & 2.32 & 0.28 & 360.04 & 16.39 & A91/3377 & $39 \mathrm{R}-1,104$ & Bulk & 2.30 & 0.15 \\
\hline 340.29 & 14.77 & A90/3783 & $37 \mathrm{R}-1.59$ & Bulk & 2.24 & 0.06 & 360.19 & 16.40 & A91/3378 & $39 \mathrm{R}-1,119$ & Bulk & 2.24 & -0.04 \\
\hline 340.49 & 14.79 & A91/3309 & $37 \mathrm{R}-1,79$ & Bulk & 2.18 & 0.00 & 360.39 & 16.42 & A91/3379 & $39 \mathrm{R}-1,139$ & Bulk & 2.32 & 0.07 \\
\hline 340.69 & 14.81 & A $91 / 3310$ & $37 \mathrm{R}-1,99$ & Bulk & 2.58 & 0.02 & 360.59 & 16.44 & A91/3380 & $39 \mathrm{R}-2,9$ & Bulk & 2.30 & 0.18 \\
\hline 340.89 & 14.82 & A91/3311 & $37 \mathrm{R}-1,119$ & Bulk & 2.25 & 0.10 & 360.79 & 16.45 & A91/3381 & $39 \mathrm{R}-2,29$ & Bulk & 2.09 & -0.02 \\
\hline 341.09 & 14.84 & A $91 / 3312$ & $37 \mathrm{R}-1,139$ & Bulk & 2.65 & 0.35 & 361.00 & 16.47 & A91/3382 & $39 \mathrm{R}-2,50$ & Bulk & 2.13 & -0.02 \\
\hline 341.29 & 14.85 & A91/3313 & $37 \mathrm{R}-2,9$ & Bulk & 2.24 & 0.16 & 361.19 & 16.49 & A90/3795 & $39 \mathrm{R}-2,69$ & Bulk & 2.28 & 0.18 \\
\hline 341.49 & 14.87 & A91/3314 & $37 \mathrm{R}-2,29$ & Bulk & 2.24 & 0.22 & 361.39 & 16.50 & A91/3383 & $39 \mathrm{R}-2,89$ & Bulk & 2.31 & 0.01 \\
\hline 341.69 & 14.89 & A91/3315 & $37 \mathrm{R}-2,49$ & Bulk & 2.65 & 0.41 & 361.57 & 16.52 & A91/3384 & $39 \mathrm{R}-2,107$ & Bulk & 2.28 & 0.10 \\
\hline 341.89 & 14.90 & A $90 / 3784$ & $37 \mathrm{R}-2,69$ & Bulk & 2.16 & 0.05 & 361.79 & 16.53 & A91/3385 & $39 \mathrm{R}-2,129$ & Bulk & 2.26 & 0.08 \\
\hline 342.09 & 14.92 & A91/3316 & $37 \mathrm{R}-2,89$ & Bulk & 2.21 & 0.29 & 361.98 & 16.55 & A91/3386 & $39 \mathrm{R}-2,148$ & Bulk & 2.24 & -0.06 \\
\hline 342.29 & 14.94 & A91/3317 & $37 \mathrm{R}-2,109$ & Bulk & 2.13 & 0.09 & 362.19 & 16.57 & A91/3387 & $39 \mathrm{R}-3,19$ & Bulk & 2.14 & -0.37 \\
\hline 342.49 & 14.95 & A91/3318 & $37 \mathrm{R}-2,129$ & Bulk & 2.22 & 0.20 & 362.39 & 16.58 & A91/3388 & $39 \mathrm{R}-3,39$ & Bulk & 2.19 & -0.12 \\
\hline 342.68 & 14.97 & A91/3319 & $37 \mathrm{R}-2,148$ & Bulk & 2.49 & 0.12 & 362.59 & 16.60 & A90/3997 & $39 \mathrm{R}-3,59$ & Bulk & 2.28 & -0.07 \\
\hline 342.89 & 14.99 & A91/3320 & $37 \mathrm{R}-3,19$ & Bulk & 2.22 & -0.25 & 362.79 & 16.62 & A91/3389 & $39 \mathrm{R}-3,79$ & Bulk & 2.28 & -0.12 \\
\hline 343.09 & 15.00 & A91/3321 & $37 \mathrm{R}-3,39$ & Bulk & 2.42 & 0.06 & 362.99 & 16.63 & A91/3390 & $39 \mathrm{R}-3,99$ & Bulk & 2.30 & -0.12 \\
\hline 343.29 & 15.02 & A90/3785 & $37 \mathrm{R}-3,59$ & Bulk & 2.42 & -0.26 & 363.19 & 16.65 & A91/3391 & $39 \mathrm{R}-3,119$ & Bulk & 2.23 & 0.04 \\
\hline 343.49 & 15.03 & A91/3325 & $37 \mathrm{R}-3,79$ & Bulk & 2.43 & 0.01 & 363.39 & 16.67 & A91/3392 & $39 \mathrm{R}-3,139$ & Bulk & 2.13 & 0.21 \\
\hline 343.69 & 15.05 & A91/3326 & $37 \mathrm{R}-3,99$ & Bulk & 2.37 & -0.02 & 363.59 & 16.68 & A91/3393 & $39 \mathrm{R}-4,9$ & Bulk & 2.18 & -0.10 \\
\hline 343.89 & 15.07 & A91/3327 & $37 \mathrm{R}-3,119$ & Bulk & 2.25 & 0.08 & 363.79 & 16.70 & A91/3394 & $39 R-4,29$ & Bulk & 2.18 & 0.01 \\
\hline 344.09 & 15.08 & A91/3328 & $37 \mathrm{R}-3,139$ & Bulk & 2.07 & 0.14 & 363.99 & 16.71 & A91/3395 & $39 R-4,49$ & Bulk & 2.09 & 0.07 \\
\hline 344.29 & 15.10 & A91/3329 & $37 \mathrm{R}-4,9$ & Bulk & 2.21 & 0.06 & 364.19 & 16.73 & A90/3998 & $39 R-4,69$ & Bulk & 2.20 & -0.15 \\
\hline 344.49 & 15.12 & A91/3330 & $37 \mathrm{R}-4,29$ & Bulk & 2.28 & 0.03 & 364.33 & 16.74 & A91/3396 & $39 \mathrm{R}-4,83$ & Bulk & 2.16 & -0.25 \\
\hline 344.69 & 15.13 & A $91 / 3331$ & $37 \mathrm{R}-4,49$ & Bulk & 2.21 & 0.03 & 364.57 & 16.76 & A91/3397 & $39 R-4,107$ & Bulk & 2.26 & -0.31 \\
\hline 344.89 & 15.15 & A90/3786 & $37 R-4,69$ & Bulk & 2.03 & 0.01 & 364.81 & 16.78 & A91/3398 & $39 \mathrm{R}-4,131$ & Bulk & 2.24 & -0.28 \\
\hline 345.09 & 15.17 & A91/3332 & $37 \mathrm{R}-4,89$ & Bulk & 2.15 & 0.29 & 364.98 & 16.80 & A91/3399 & $39 \mathrm{R}-4,148$ & Bulk & 2.01 & -0.15 \\
\hline 345.29 & 15.18 & A91/3333 & $37 \mathrm{R}-4,109$ & Bulk & 2.26 & 0.16 & 365.19 & 16.81 & A91/3400 & $39 \mathrm{R}-5,19$ & Bulk & 2.21 & 0.00 \\
\hline 345,49 & 15.20 & A91/3334 & $37 \mathrm{R}-4,129$ & Bulk & 2.30 & 0.28 & 365.39 & 16.83 & A91/3401 & $39 \mathrm{R}-5,39$ & Bulk & 2.23 & 0.13 \\
\hline 345.68 & 15.21 & A91/3335 & $37 \mathrm{R}-4,148$ & Bulk & 2.25 & 0.27 & 365.59 & 16.85 & A90/3999 & $39 \mathrm{R}-5,59$ & Bulk & 2.37 & 0.19 \\
\hline 345.89 & 15.23 & A91/3336 & $37 \mathrm{R}-5,19$ & Bulk & 2.27 & 0.07 & 365.79 & 16.86 & A91/3402 & $39 \mathrm{R}-5,79$ & Bulk & 2.33 & 0.21 \\
\hline 346.09 & 15.25 & A91/3337 & $37 \mathrm{R}-5,39$ & Bulk & 2.30 & 0.22 & 365.99 & 16.88 & A91/3403 & $39 \mathrm{R}-5,99$ & Bulk & 2.37 & 0.20 \\
\hline 346.49 & 15.28 & A91/3338 & $37 \mathrm{R}-5,79$ & Bulk & 2.63 & 0.17 & 366.19 & 16.89 & A91/3404 & $39 \mathrm{R}-5,119$ & Bulk & 2.37 & -0.04 \\
\hline 346.69 & 15.30 & A91/3398 & $37 \mathrm{R}-5,99$ & Bulk & 2.46 & 0.10 & 366.39 & 16.91 & A91/3405 & $39 \mathrm{R}-5,139$ & Bulk & 2.31 & 0.19 \\
\hline 346.89 & 15.31 & A $91 / 3340$ & $37 \mathrm{R}-5,119$ & Bulk & 2.40 & 0.19 & 366.59 & 16.93 & A91/3406 & $39 \mathrm{R}-6,9$ & Bulk & 2.31 & 0.03 \\
\hline 347.09 & 15.33 & A91/3342 & $37 \mathrm{R}-5,139$ & Bulk & 2.27 & -0.18 & 366.79 & 16.94 & A91/3407 & $39 \mathrm{R}-6,29$ & Bulk & 2.19 & -0.02 \\
\hline 347.29 & 15.35 & A91/3343 & $37 \mathrm{R}-6,9$ & Bulk & 2.39 & -0.10 & 366.99 & 16.96 & A91/3408 & $39 \mathrm{R}-6,49$ & Bulk & 2.13 & 0.01 \\
\hline 347.49 & 15.36 & A91/3344 & $37 \mathrm{R}-6,29$ & Bulk & 2.15 & 0.04 & 367.19 & 16.98 & A90/4000 & $39 \mathrm{R}-6,69$ & Bulk & 2.36 & -0.01 \\
\hline 349.59 & 15.53 & A91/3345 & $38 \mathrm{R}-1,19$ & Bulk & 2.59 & 0.07 & 367.39 & 16.99 & A91/3409 & $39 \mathrm{R}-6,89$ & Bulk & 2.35 & -0.24 \\
\hline 349.79 & 15.55 & A91/3346 & $38 \mathrm{R}-1,39$ & Bulk & 2.75 & 0.31 & 367.59 & 17.01 & A91/3410 & $39 \mathrm{R}-6,109$ & Bulk & 2.26 & -0.26 \\
\hline 349.99 & 15.57 & A90/3789 & $38 \mathrm{R}-1,59$ & Bulk & 2.59 & -0.12 & 368.89 & 17.12 & A91/3411 & $40 X-1,19$ & Bulk & 2.21 & 0.03 \\
\hline 350.19 & 15.58 & A91/3347 & $38 \mathrm{R}-1,79$ & Bulk & 2.40 & 0.03 & 369.09 & 17.13 & A $91 / 3412$ & $40 X-1,39$ & Bulk & 2.35 & -0.03 \\
\hline 350.39 & 15.60 & A91/3348 & $38 \mathrm{R}-1,99$ & Bulk & 2.63 & 0.06 & 369.49 & 17.17 & A91/3413 & $40 X-1,79$ & Bulk & 2.28 & -0.39 \\
\hline 350.59 & 15.62 & A91/3349 & $38 \mathrm{R}-1,119$ & Bulk & 2.68 & 0.18 & 370.89 & 17.28 & A $90 / 4002$ & $40 X-2,69$ & Bulk & 2.30 & 0.17 \\
\hline 350.77 & 15.63 & A91/3350 & $38 \mathrm{R}-1,137$ & Bulk & 2.66 & 0.03 & 372.29 & 17.39 & A90/4003 & $40 X-3,59$ & Bulk & 2.44 & 0.28 \\
\hline 350.99 & 15.65 & A91/3351 & $38 \mathrm{R}-2,9$ & Bulk & 2.41 & -0.13 & 373.90 & 17.53 & A90/4004 & $40 X-4,70$ & Bulk & 2.29 & 0.00 \\
\hline 351.19 & 15.67 & A $91 / 3352$ & $38 \mathrm{R}-2,29$ & Bulk & 2.26 & 0.00 & 375.29 & 17.64 & A90/4005 & $40 X-5,59$ & Bulk & 2.08 & -0.16 \\
\hline 351.39 & 15.68 & A $91 / 3353$ & $38 \mathrm{R}-2,49$ & Bulk & 2.43 & 0.18 & 376.69 & 17.76 & A90/4006 & $40 X-6,49$ & Bulk & 2.17 & -0.21 \\
\hline 351.59 & 15.70 & A $90 / 3790$ & $38 \mathrm{R}-2,69$ & Bulk & 2.75 & 0.15 & 378.88 & 17.93 & A90/4007 & $41 \mathrm{R}-1,58$ & Bulk & 2.25 & 0.26 \\
\hline 351.79 & 15.71 & A91/3354 & $38 \mathrm{R}-2,89$ & Bulk & 2.82 & 0.16 & 380.31 & 18.05 & A90/4008 & $41 R-2,51$ & Bulk & 2.06 & 0.05 \\
\hline 351.99 & 15.73 & A91/3355 & $38 \mathrm{R}-2,109$ & Bulk & 2.74 & 0.15 & 381.89 & 18.18 & A90/4009 & $41 \mathrm{R}-3,59$ & Bulk & 2.14 & 0.43 \\
\hline 352.19 & 15.75 & A91/3356 & $38 \mathrm{R}-2,129$ & Bulk & 2.56 & 0.41 & 383.48 & 18.31 & A $90 / 4010$ & $41 R-4,68$ & Bulk & 1.90 & 0.40 \\
\hline 352.38 & 15.76 & A91/3357 & $38 \mathrm{R}-2,148$ & Bulk & 2.52 & 0.37 & 384.89 & 18.43 & A90/4011 & $4 I R-5,59$ & Bulk & 1.56 & 0.28 \\
\hline 352.59 & 15.78 & A91/3358 & $38 \mathrm{R}-3,19$ & Bulk & 2.61 & 0.09 & & & & & & & \\
\hline
\end{tabular}


APPENDIX B

Bulk-rock and Foraminiferal Isotope Data from Hole 806B

\begin{tabular}{|c|c|c|c|c|c|c|c|c|c|c|c|c|c|}
\hline $\begin{array}{l}\text { Depth } \\
\text { (mbsf) }\end{array}$ & $\begin{array}{l}\text { Age } \\
(\mathrm{Ma})\end{array}$ & Lab no. & $\begin{array}{l}\text { Core, section, } \\
\text { interval }(\mathrm{cm})\end{array}$ & Species & $\delta^{13} \mathrm{C}$ & $\delta^{18} \mathrm{O}$ & $\begin{array}{l}\text { Depth } \\
\text { (mbsf) }\end{array}$ & $\begin{array}{l}\text { Age } \\
\text { (Ma) }\end{array}$ & Lab no. & $\begin{array}{l}\text { Core, section, } \\
\text { interval }(\mathrm{cm})\end{array}$ & Species & $\delta^{13} \mathrm{C}$ & $\delta^{18} \mathrm{O}$ \\
\hline 416.99 & 12.51 & A90/2941 & $45 X-1,9$ & Bulk & 1.44 & -0.21 & 422.19 & 12.69 & A91/1449 & $45 X-4,79$ & Cib. spp. & 1.01 & 2.23 \\
\hline 417.19 & 12.52 & A91/682 & $45 X-1,29$ & D. alt. & 1.97 & -0.82 & 422.39 & 12.70 & A91/416 & $45 X-4,99$ & G. venez & 1.88 & -0.82 \\
\hline 417.19 & 12.52 & A90/2942 & $45 X-1,29$ & Bulk & 1.56 & 0.24 & 422.39 & 12.70 & A91/1450 & $45 X-4,99$ & Cib. spp. & 1.11 & 2.34 \\
\hline 417.39 & 12.53 & A91/681 & $45 X-1,49$ & G. venez. & 1.64 & -0.69 & 422.39 & 12.70 & A90/2965 & $45 X-4,99$ & Bulk & 1.52 & 0.16 \\
\hline 417.39 & 12.53 & A90/2943 & $45 X-1,49$ & Bulk & 1.62 & -0.02 & 422.39 & 12.70 & A91/415 & $45 X-4,99$ & D. alt. & 2.14 & -0.88 \\
\hline 417.39 & 12.53 & A91/680 & $45 X-1,49$ & D. alt. & 1.99 & -0.99 & 422.59 & 12.70 & A91/1451 & $45 X-4,119$ & P. wuell. & 1.06 & 1.96 \\
\hline 417.59 & 12.53 & A91/1763 & $45 X-1,69$ & D. alt. & 2.13 & -1.23 & 422.59 & 12.70 & A90/2966 & $45 X-4,119$ & Bulk & 1.63 & 0.09 \\
\hline 417.59 & 12.53 & A91/2405 & $45 X-1,69$ & G. venez & 1.81 & -0.64 & 422.59 & 12.70 & A91/411 & $45 X-4,119$ & G. venez. & 2.29 & -0.96 \\
\hline 417.59 & 12.53 & A90/2944 & $45 X-1,69$ & Bulk & 1.64 & -0.10 & 422.59 & 12.70 & A91/410 & $45 X-4,119$ & D. alt. & 2.05 & -0.97 \\
\hline 417.79 & 12.54 & A90/2945 & $45 X-1,89$ & Bulk & 1.51 & 0.05 & 422.59 & 12.70 & A91/1452 & $45 X-4,119$ & Cib. spp. & 1.12 & 2.22 \\
\hline 417.79 & 12.54 & A91/674 & $45 X-1,89$ & D. alt. & 2.14 & -1.15 & 422.79 & 12.71 & A90/2967 & $45 X-4,129$ & Bulk & 1.57 & 0.04 \\
\hline 417.79 & 12.54 & A91/675 & $45 X-1,89$ & $G$, venez. & 1.63 & -0.77 & 422.79 & 12.71 & A91/1453 & $45 X-4,139$ & Cib. spp. & 1.03 & 2.16 \\
\hline 417.99 & 12.55 & A90/2946 & $45 X-1,109$ & Bulk & 1.43 & 0.62 & 422.79 & 12.71 & A91/405 & $45 X-4,139$ & D. alt. & 2.08 & -0.87 \\
\hline 417.99 & 12.55 & A91/673 & $45 \mathrm{X}-1,109$ & G. venez. & 1.41 & -0.61 & 422.79 & 12.71 & A91/406 & $45 X-4,139$ & G. venez. & 2.03 & -0.86 \\
\hline 417.99 & 12.55 & A91/672 & $45 X-1,109$ & D. alt. & 2.14 & -0.91 & 422.99 & 12.72 & A91/1454 & $45 X-5,9$ & Cib. spp. & 1.00 & 2.14 \\
\hline 418.19 & 12.55 & A90/2947 & $45 X-1,129$ & Bulk & 1.36 & -0.14 & 422.99 & 12.72 & A90/2968 & $45 X-5,9$ & Bulk & 1.56 & 0.06 \\
\hline 418.19 & 12.55 & A91/670 & $45 X-1,129$ & D. alt. & 1.94 & -1.14 & 422.99 & 12.72 & A91/401 & $45 X-5,9$ & G. venez. & 1.66 & -0.72 \\
\hline 418.19 & 12.55 & A91/671 & $45 X-1,129$ & G. venez. & 1.83 & -0.77 & 422.99 & 12.72 & A91/400 & $45 X-5,9$ & D. alt. & 2.28 & -1.07 \\
\hline 418.59 & 12.57 & A90/2949 & $45 X-2,19$ & Bulk & 1.31 & 0.20 & 423.19 & 12.72 & A91/254 & $45 X-5,29$ & D. alt. & 2.15 & -0.88 \\
\hline 418.79 & 12.57 & A $90 / 2950$ & $45 X-2,39$ & Bulk & 1.35 & 0.14 & 423.19 & 12.72 & A91/1457 & $45 X-5,29$ & Cib. spp. & 0.87 & 2.11 \\
\hline 418.99 & 12.58 & A91/1761 & $45 X-2,59$ & D. alt. & 1.88 & -0.96 & 423.19 & 12.72 & A90/2969 & $45 X-5,29$ & Bulk & 1.59 & -0.14 \\
\hline 418.99 & 12.58 & A90/2951 & $45 X-2,59$ & Bulk & 1.45 & 0.21 & 423.19 & 12.72 & A91/255 & $45 X-5,29$ & G. venez & 1.92 & -0.62 \\
\hline 418.99 & 12.58 & A91/1427 & $45 X-2,59$ & Cib. spp. & 0.85 & 2.13 & 423.39 & 12.73 & A91/1456 & $45 X-5,49$ & Cib. spp. & 1.03 & 2.26 \\
\hline 418.99 & 12.58 & A91/1426 & $45 X-2,59$ & $P$ wuell. & 0.97 & 1.99 & 423.39 & 12.73 & A91/259 & $45 X-5,49$ & D. alt. & 2.19 & -0.61 \\
\hline 418.99 & 12.58 & A91/1762 & $45 \times-2,59$ & $G$. venez & 1.49 & -0.20 & 423.39 & 12.73 & A90/2970 & $45 X-5,49$ & Bulk & 1.63 & -0.02 \\
\hline 419.19 & 12.59 & A91/1428 & $45 X-2,79$ & IB & 0.86 & 2.26 & 423.39 & 12.73 & A $91 / 260$ & $45 X-5,49$ & G. venez & 1.71 & -0.32 \\
\hline 419.19 & 12.59 & A90/2952 & $45 X-2,79$ & Bulk & 1.45 & 0.10 & 423.39 & 12.73 & A91/1455 & $45 X-5,49$ & P. wuell. & 1.02 & 2.24 \\
\hline 419.39 & 12.59 & A91/1429 & $45 X-2,99$ & Cib. spp. & 1.00 & 2.28 & 423.59 & 12.74 & A91/1756 & $45 X-5,69$ & G. venez. & 1.75 & -0.04 \\
\hline 419.39 & 12.59 & A $90 / 2953$ & $45 X-2,99$ & Bulk & 1.47 & 0.26 & 423.59 & 12.74 & A90/2971 & $45 X-5,69$ & Bulk & 1.50 & 0.23 \\
\hline 419.59 & 12.60 & A91/1431 & $45 X-2,119$ & Cib. spp. & 0.82 & 2.24 & 423.59 & 12.74 & A91/1458 & $45 X-5,69$ & Cib. spp. & 1.11 & 2.27 \\
\hline 419.59 & 12.60 & A90/2954 & $45 X-2,119$ & Bulk & 1.48 & 0.22 & 423.59 & 12.74 & A91/1755 & $45 X-5,69$ & D. alt. & 2.13 & -1.00 \\
\hline 419.59 & 12.60 & A91/1430 & $45 X-2,119$ & P. wuell. & 1.08 & 2.18 & 423.79 & 12.74 & A91/245 & $45 X-5,89$ & G. venez. & 1.79 & -0.38 \\
\hline 419.79 & 12.61 & A91/1432 & $45 X-2,139$ & $P$ wuell. & 1.00 & 2.13 & 423.79 & 12.74 & A91/1459 & $45 X-5,89$ & Cib. spp. & 1.06 & 2.18 \\
\hline 419.79 & 12.61 & A91/1433 & $45 X-2,139$ & Cib. spp. & 0.92 & 2.11 & 423.79 & 12.74 & A91/244 & $45 X-5,89$ & D. alt. & 2.13 & -1.01 \\
\hline 419.79 & 12.61 & A91/575 & $45 X-2,139$ & G. venez. & 1.71 & -0.51 & 423.79 & 12.74 & A90/2972 & $45 X-5,89$ & Bulk & 1.59 & 0.19 \\
\hline 419.79 & 12.61 & A90/2948 & $45 X-2,139$ & Bulk & 1.47 & 0.24 & 423.99 & 12.75 & A90/2973 & $45 X-5,109$ & Bulk & 1.52 & -0.11 \\
\hline 419.99 & 12.61 & A90/2955 & $45 X-3,9$ & Bulk & 1.49 & -0.02 & 423.99 & 12.75 & A91/1460 & $45 X-5,109$ & P. wuell. & 1.08 & 2.12 \\
\hline 419.99 & 12.61 & A91/569 & $45 X-3,9$ & D. alt. & 2.01 & -1.10 & 423.99 & 12.75 & A91/239 & $45 X-5,109$ & D. alt. & 2.14 & -0.92 \\
\hline 419.99 & 12.61 & A91/1434 & $45 X-3,9$ & P. wuell. & 1.07 & 1.98 & 423.99 & 12.75 & A91/240 & $45 X-5,109$ & $G$. venez. & 1.88 & -0.76 \\
\hline 419.99 & 12.61 & A91/1435 & $45 X-3,9$ & Cib. spp. & 1.03 & 2.01 & 423.99 & 12.75 & A91/1461 & $45 X-5,109$ & Cib. spp. & 1.00 & 2.21 \\
\hline 419.99 & 12.61 & A91/570 & $45 X-3,9$ & G. venez. & 1.97 & -0.74 & 424.19 & 12.76 & A91/1463 & $45 X-5,129$ & Cib. spp. & 1.06 & 2.15 \\
\hline 420.19 & 12.62 & A90/2956 & $45 X-3,29$ & Bulk & 1.46 & -0.01 & 424.19 & 12.76 & A90/2974 & $45 X-5,129$ & Bulk & 1.45 & -0.08 \\
\hline 420.19 & 12.62 & A $91 / 460$ & $45 \times-3,29$ & D. alt. & 2.17 & -0.97 & 424.19 & 12.76 & A91/235 & $45 X-5,129$ & G. venez. & 1.70 & -0.90 \\
\hline 420.39 & 12.63 & A91/1437 & $45 X-3,49$ & Cib. spp. & 0.98 & 2.08 & 424.19 & 12.76 & A91/234 & $45 X-5,129$ & D. alt. & 1.91 & -0.95 \\
\hline 420.39 & 12.63 & A91/455 & $45 X-3,49$ & D. alt. & 1.90 & -0.59 & 424.19 & 12.76 & A91/1462 & $45 X-5,129$ & $P$ wuell. & 1.10 & 2.28 \\
\hline 420.39 & 12.63 & A91/456 & $45 X-3,49$ & G. venez & 1.95 & -0.77 & 424.59 & 12.77 & A $91 / 230$ & $45 X-6,19$ & G. venez. & 1.81 & -0.82 \\
\hline 420.39 & 12,63 & A90/2957 & $45 X-3,49$ & Bulk & 1.46 & 0.04 & 424.59 & 12.77 & A90/2975 & $45 X-6,19$ & Bulk & 1.53 & 0.30 \\
\hline 420.39 & 12.63 & A91/1436 & $45 X-3,49$ & P. wuell. & 1.06 & 2.17 & 424.59 & 12.77 & A91/229 & $45 X-6,19$ & D. alt. & 1.87 & -1.06 \\
\hline 420.59 & 12.63 & A91/1438 & $45 X-3,69$ & P. wuell. & 0.91 & 2.09 & 424.59 & 12.77 & A91/1464 & $46 \times-6,19$ & Cib. spp. & 0.96 & 1.92 \\
\hline 420.59 & 12.63 & A91/1439 & $45 X-3,69$ & Cib. spp. & 0.96 & 2.17 & 424.79 & 12.78 & A90/2976 & $45 X-6,39$ & Bulk & 1.35 & -0.14 \\
\hline 420.59 & 12.63 & A91/1759 & $45 X-3,69$ & D. alt. & 1.95 & -0.99 & 424.79 & 12.78 & A91/186 & $45 X-6,39$ & D. alt. & 1.96 & -0.93 \\
\hline 420.59 & 12.63 & A $90 / 2958$ & $45 X-3,69$ & Bulk & 1.47 & 0.17 & 424.79 & 12.78 & A91/1465 & $45 X-6,39$ & Cib. spp. & 0.98 & 1.91 \\
\hline 420.59 & 12.63 & A91/1760 & $45 X-3,69$ & G. venez. & 1.79 & -0.71 & 424.79 & 12.78 & A91/187 & $45 X-6,39$ & $G$. venez & 1.92 & -0.77 \\
\hline 420.79 & 12.64 & A91/1440 & $45 X-3,89$ & P. wuell. & 1.08 & 2.21 & 424.99 & 12.78 & A91/1466 & $45 X-6,59$ & $P$ wuell. & 1.02 & 2.25 \\
\hline 420.79 & 12.64 & A91/1441 & $45 X-3,89$ & Cib. spp. & 0.74 & 2.27 & 424.99 & 12.78 & A91/182 & $45 X-6,59$ & G. venez & 1.74 & -0.49 \\
\hline 420.79 & 12.64 & A90/2959 & $45 X-3,89$ & Bulk & 1.49 & -0.05 & 424.99 & 12.78 & A91/1467 & $45 X-6,59$ & Cib. spp. & 1.07 & 2.09 \\
\hline 420.79 & 12.64 & A91/446 & $45 X-3,89$ & G. venez & 1.65 & -0.48 & 424.99 & 12.78 & A91/1753 & $45 X-6,59$ & D. alt. & 1.83 & -1.22 \\
\hline 420.79 & 12.64 & A91/445 & $45 X-3,89$ & D. ait. & 2.06 & -0.77 & 424.99 & 12.78 & A90/2977 & $45 X-6,59$ & Bulk & 1.41 & -0.24 \\
\hline 420.99 & 12.65 & A $91 / 440$ & $45 X-3,109$ & D. ait. & 2.00 & -0.86 & 425.19 & 12.79 & A90/2978 & $45 X-6,79$ & Bulk & 1.47 & -0.07 \\
\hline 420.99 & 12.65 & A $90 / 2960$ & $45 X-3,109$ & Bulk & 1.54 & 0.17 & 425.19 & 12.79 & A91/177 & $45 X-6,79$ & G. venez & 1.92 & -0.76 \\
\hline 420.99 & 12.65 & A $91 / 1442$ & $45 X-3,109$ & Cib. spp. & 0.85 & 2.13 & 425.19 & 12.79 & A91/176 & $45 X-6,79$ & D. alt. & 1.87 & -0.74 \\
\hline 420.99 & 12.65 & A91/441 & $45 X-3,109$ & G. venez. & 1.89 & -0.37 & 425.19 & 12.79 & A91/1468 & $45 X-6,79$ & Cib. spp. & 0.97 & 2.06 \\
\hline 421.59 & 12.67 & A91/436 & $45 X-4,19$ & G. venez & 2.16 & -0.93 & 425.39 & 12.80 & A91/1469 & $45 X-6,99$ & P. wuell. & 1.11 & 2.02 \\
\hline 421.59 & 12.67 & A91/1443 & $45 \times-4,19$ & Cib. spp. & 0.90 & 2.38 & 425.39 & 12.80 & A90/2979 & $45 X-6,99$ & Bulk & 1.53 & -0.10 \\
\hline 421.59 & 12.67 & A91/435 & $45 \times-4,19$ & D. alt. & 2.07 & -0.65 & 425.39 & 12.80 & A $91 / 1470$ & $45 X-6,99$ & Cib. spp. & 1.12 & 2.06 \\
\hline 421.59 & 12.67 & A90/2961 & $45 \times-4,19$ & Bulk & 1.46 & 0.36 & 425.39 & 12.80 & A91/172 & $45 X-6,99$ & G. venez. & 1.92 & -0.90 \\
\hline 421.79 & 12.68 & A91/1444 & $45 \times-4,39$ & $P$ wuell. & 0.99 & 2.27 & 425.39 & 12.80 & A91/171 & $45 X-6,99$ & D. alt. & 1.95 & -0.89 \\
\hline 421.79 & 12.68 & A91/431 & $45 X-4,39$ & G. venez & 1.76 & -0.81 & 425.59 & 12.80 & A90/2980 & $45 X-6,119$ & Bulk & 1.43 & -0.02 \\
\hline 421.79 & 12.68 & A $90 / 2962$ & $45 \times-4,39$ & Bulk & 1.48 & 0.12 & 425.79 & 12.80 & A91/676 & $45 X-6,139$ & D. alt. & 1.98 & -0.99 \\
\hline 421.79 & 12.68 & A91/430 & $45 X-4,39$ & D. alt. & 2.05 & -0.69 & 425.79 & 12.80 & A91/1471 & $45 X-6,139$ & Cib. spp. & 0.96 & 2.12 \\
\hline 421.79 & 12.68 & A91/1445 & $45 \times-4,39$ & Cib. spp. & 0.97 & 2.36 & 425.79 & 12.80 & A91/677 & $45 X-6,139$ & G. venez. & 1.41 & 0.08 \\
\hline 421.99 & 12.68 & A $90 / 2963$ & $45 \times-4,59$ & Bulk & 1.41 & 0.06 & 425.79 & 12.80 & A90/2981 & $45 X-6,139$ & Bulk & 1.46 & 0.11 \\
\hline 421.99 & 12.68 & A91/1447 & $45 \times-4,59$ & Cib. spp. & 1.02 & 2.22 & 426.59 & 12.81 & A91/162 & $46 \mathrm{X}-1,9$ & G. venez & 1.59 & 0.12 \\
\hline 421.99 & 12.68 & A91/426 & $45 \times-4,59$ & G. venez & 1.91 & -0.54 & 426.59 & 12.81 & A $91 / 161$ & $46 \mathrm{X}-1,9$ & D. alt. & 1.94 & -0.71 \\
\hline 421.99 & 12.68 & A91/1757 & $45 \times-4,59$ & D. alt. & 1.87 & -0.85 & 426.59 & 12.81 & A90/2982 & $46 \mathrm{X}-1,9$ & Bulk & 1.85 & 0.39 \\
\hline 421.99 & 12.68 & A91/1446 & $45 X-4,59$ & P. wuell. & 1.04 & 2.30 & 426.79 & 12.81 & A $90 / 2983$ & $46 \mathrm{X}-1,29$ & Bulk & 1.79 & 0.33 \\
\hline 422.19 & 12.69 & A91/1448 & $45 \times-4,79$ & $P$ wuell. & 1.12 & 2.05 & 426.79 & 12.81 & A91/156 & $46 \mathrm{X}-1,29$ & D. alt. & 1.87 & -0.86 \\
\hline 422.19 & 12.69 & A90/2964 & $45 \times-4,79$ & Bulk & 1.39 & -0.06 & 426.79 & 12.81 & A91/157 & $46 \mathrm{X}-1,29$ & G. venez & 1.87 & -1.00 \\
\hline
\end{tabular}


Appendix B (continued).

\begin{tabular}{|c|c|c|c|c|c|c|c|c|c|c|c|c|c|}
\hline $\begin{array}{l}\text { Depth } \\
\text { (mbsf) }\end{array}$ & $\begin{array}{l}\text { Age } \\
(\mathrm{Ma})\end{array}$ & Lab no. & $\begin{array}{l}\text { Core, section, } \\
\text { interval }(\mathrm{cm})\end{array}$ & Species & $\delta^{13} \mathrm{C}$ & $\delta^{18} \mathrm{O}$ & $\begin{array}{l}\text { Depth } \\
\text { (mbsf) }\end{array}$ & $\begin{array}{l}\text { Age } \\
\text { (Ma) }\end{array}$ & Lab no. & $\begin{array}{l}\text { Core, section, } \\
\text { interval }(\mathrm{cm})\end{array}$ & Species & $\delta^{13} \mathrm{C}$ & $\delta^{18} \mathrm{O}$ \\
\hline 426.99 & 12.81 & A91/152 & $46 X-1,49$ & G. venez. & 2.27 & -1.01 & 431.99 & 12.85 & A90/4681 & $46 X-4,99$ & G. venez. & 2.04 & -0.89 \\
\hline 426.99 & 12.81 & A91/1473 & $46 X-1,49$ & P. wuell. & 1.06 & 2.10 & 431.99 & 12.85 & A91/1288 & $46 \times-4,99$ & Cib. mun. & 0.80 & 2.07 \\
\hline 426.99 & 12.81 & A90/2984 & $46 \mathrm{X}-1,49$ & Bulk & 1.88 & 0.52 & 431.99 & 12.85 & A $90 / 4680$ & $46 \times-4,99$ & D. alt. & 1.97 & -1.03 \\
\hline 426.99 & 12.81 & A91/151 & $46 \mathrm{X}-1,49$ & D. alt. & 1.88 & -0.89 & 431.99 & 12.85 & A91/3180 & $46 X-4,99$ & Bulk & 1.58 & 0.42 \\
\hline 427.19 & 12.81 & A91/1752 & $46 X-1,69$ & G. venez. & 1.95 & -0.84 & 432.19 & 12.85 & A91/3181 & $46 X-4,119$ & Bulk & 1.61 & 0.23 \\
\hline 427.19 & 12.81 & A91/1474 & $46 X-1,69$ & Cib. spp. & 1.08 & 1.97 & 432.19 & 12.85 & A90/4675 & $46 X-4,119$ & D. alt. & 1.90 & -0.99 \\
\hline 427.19 & 12.81 & A91/1751 & $46 X-1,69$ & D. alt. & 2.07 & -0.67 & 432.19 & 12.85 & A91/1292 & $46 X-4,119$ & Cib. mun. & 0.87 & 2.09 \\
\hline 427.19 & 12.81 & A90/2985 & $46 \mathrm{X}-1,69$ & Bulk & 1.94 & 0.34 & 432.19 & 12.85 & A90/4676 & $46 X-4,119$ & G. venez. & 2.03 & -1.21 \\
\hline 427.39 & 12.82 & A90/2986 & $46 X-1,89$ & Bulk & 1.84 & 0.33 & 432.23 & 12.85 & A91/3182 & $46 X-4,123$ & Bulk & 1.58 & 0.35 \\
\hline 427.39 & 12.82 & A90/4922 & $46 X-1,89$ & D. alt. & 2.13 & -0.98 & 432.39 & 12.85 & A90/4670 & $46 \times-4,139$ & D. alt. & 2.05 & -0.92 \\
\hline 427.39 & 12.82 & A91/1475 & $46 \mathrm{X}-1,89$ & P. wuell. & 1.17 & 2.12 & 432.39 & 12.85 & A $91 / 3183$ & $46 \times-4,139$ & Bulk & 1.61 & 0.29 \\
\hline 427.39 & 12.82 & A91/1476 & $46 X-1,89$ & Cib. spp. & 1.05 & 2.07 & 432.39 & 12.85 & A $90 / 4671$ & $46 X-4,139$ & G. venez. & 1.76 & -0.77 \\
\hline 427.39 & 12.82 & A90/4923 & $46 \mathrm{X}-1,89$ & G. venez. & 1.87 & -0.60 & 432.59 & 12.86 & A90/4637 & $46 X-5,9$ & G. venez. & 1.93 & -0.83 \\
\hline 427.59 & 12.82 & A90/4918 & $46 \mathrm{X}-1,109$ & G. venez & 2.11 & -0.95 & 432.59 & 12.86 & A91/3184 & $46 X-5,9$ & Bulk & 1.51 & 0.02 \\
\hline 427.59 & 12.82 & A91/1477 & $46 \mathrm{X}-1,109$ & P. wuell. & 1.02 & 2.15 & 432.59 & 12.86 & A90/4636 & $46 X-5,9$ & D. alt. & 2.05 & -0.96 \\
\hline 427.59 & 12.82 & A90/4917 & $46 \mathrm{X}-1,109$ & D. alt. & 1.98 & -0.95 & 432.79 & 12.86 & A90/4632 & $46 X-5,29$ & G. venez. & 2.01 & -0.95 \\
\hline 427.59 & 12.82 & A90/2987 & $46 \mathrm{X}-1,109$ & Bulk & 1.80 & 0.37 & 432.79 & 12.86 & A91/3185 & $46 X-5,29$ & Bulk & 1.65 & 0.28 \\
\hline 427.79 & 12.82 & A91/1478 & $46 \mathrm{X}-1,129$ & Cib. spp. & 0.98 & 2.20 & 432.79 & 12.86 & A90/4631 & $46 X-5,29$ & D. alt. & 2.15 & -0.97 \\
\hline 427.79 & 12.82 & A90/4913 & $46 \mathrm{X}-1,129$ & G. venez & 1.87 & -0.60 & 432.99 & 12.86 & A91/3189 & $46 X-5,49$ & Bulk & 1.70 & 0.35 \\
\hline 427.79 & 12.82 & A90/2988 & $46 \mathrm{X}-1,129$ & Bulk & 1.69 & 0.22 & 432.99 & 12.86 & A90/4627 & $46 \times-5,49$ & G. venez. & 2.21 & -0.77 \\
\hline 427.79 & 12.82 & A90/4912 & $46 \mathrm{X}-1,129$ & D. alt. & 1.80 & -0.76 & 432.99 & 12.86 & A90/4626 & $46 X-5,49$ & D. alt. & 2.22 & -0.80 \\
\hline 428.19 & 12.82 & A90/4907 & $46 \mathrm{X}-2,19$ & G. venez & 1.89 & -0.80 & 433.19 & 12.86 & A $91 / 3190$ & $46 X-5,69$ & Bulk & 1.72 & 0.37 \\
\hline 428.19 & 12.82 & A90/4906 & $46 \mathrm{X}-2,19$ & D. alt. & 1.80 & -1.00 & 433.19 & 12.86 & A91/1744 & $46 X-5,69$ & G. venez. & 2.06 & -0.73 \\
\hline 428.19 & 12.82 & A91/1479 & $46 X-2,19$ & Cib. spp. & 0.84 & 1.97 & 433.19 & 12.86 & A91/1743 & $46 X-5,69$ & D. alt. & 2.27 & -1.01 \\
\hline 428.19 & 12.82 & A90/2989 & $46 \times-2,19$ & Bulk & 1.57 & 0.50 & 433.39 & 12.86 & A90/4616 & $46 X-5,89$ & D. alt. & 2.27 & -0.96 \\
\hline 428.39 & 12.82 & A90/2990 & $46 \times-2,39$ & Bulk & 1.49 & 0.41 & 433.39 & 12.86 & A91/3186 & $46 X-5,89$ & Bulk & 1.77 & 0.29 \\
\hline 428.39 & 12.82 & A $91 / 1480$ & $46 X-2,59$ & Cib. spp. & 0.86 & 2.17 & 433.39 & 12.86 & A90/4617 & $46 X-5,89$ & G. venez & 2.23 & -0.83 \\
\hline 428.59 & 12.82 & A91/1749 & $46 \mathrm{X}-2,59$ & D. alt. & 1.85 & -1.12 & 433.59 & 12.86 & A91/3187 & $46 X-5,109$ & Bulk & 1.74 & 0.40 \\
\hline 428.59 & 12.82 & A90/2991 & $46 X-2,59$ & Bulk & 1.53 & 0.27 & 433.59 & 12.86 & A90/4612 & $46 X-5,109$ & G. venez. & 2.26 & -0.83 \\
\hline 428.59 & 12.82 & A91/1750 & $46 \times-2,59$ & G. venez. & 1.58 & -0.10 & 433.59 & 12.86 & A90/4611 & $46 X-5,109$ & D. alt. & 2.29 & -0.86 \\
\hline 428.79 & 12.83 & A90/4893 & $46 X-2,79$ & G. venez & 1.77 & -0.62 & 433.79 & 12.87 & A91/3188 & $46 X-5,129$ & Bulk & 1.80 & 0.42 \\
\hline 428.79 & 12.83 & A91/1281 & $46 X-2,79$ & Cib. & 0.59 & 2.18 & 433.79 & 12.87 & A90/4606 & $46 \times-5,129$ & D. alt. & 2.07 & -0.87 \\
\hline 428.79 & 12.83 & A90/2992 & $46 X-2,79$ & Bulk & 1.45 & 0.13 & 433.79 & 12.87 & A91/1290 & $46 X-5,129$ & Cib. mun. & 1.13 & 2.19 \\
\hline 428.79 & 12.83 & A90/4892 & $46 X-2,79$ & D. alt. & 2.09 & -0.98 & 433.79 & 12.87 & A90/4607 & $46 X-5,129$ & $G$, venez. & 2.10 & -0.84 \\
\hline 428.99 & 12.83 & A90/4886 & $46 X-2,99$ & G. venez. & 1.73 & -0.40 & 434.19 & 12.87 & A91/3191 & $46 \mathrm{X}-6,19$ & Bulk & 1.75 & 0.30 \\
\hline 428.99 & 12.83 & A90/2993 & $46 \times-2,99$ & Bulk & 1.65 & 0.27 & 434.19 & 12.87 & A90/4601 & $46 \times-6,19$ & D. alt. & 2.11 & -0.98 \\
\hline 428.99 & 12.83 & A91/1285 & $46 \mathrm{X}-2,99$ & Cib. mun. & 1.06 & 2.26 & 434.19 & 12.87 & A90/4602 & $46 \times-6,19$ & G. venez. & 1.87 & -0.86 \\
\hline 428.99 & 12.83 & A90/4885 & $46 \mathrm{X}-2,99$ & D. alt. & 1.99 & -1.03 & 434.19 & 12.87 & A91/1291 & $46 \mathrm{X}-6,19$ & Cib. mun. & 1.07 & 2.25 \\
\hline 429.19 & 12.83 & A90/4880 & $46 X-2,119$ & G. venez. & 1.73 & -0.61 & 434.39 & 12.87 & A91/3192 & $46 \mathrm{X}-6,39$ & Bulk & 1.68 & 0.35 \\
\hline 429.19 & 12.83 & A90/2994 & $46 \mathrm{X}-2,119$ & Bulk & 1.74 & 0.33 & 434.39 & 12.87 & A90/4546 & $46 \mathrm{X}-6,39$ & G. venez & 2.23 & -0.91 \\
\hline 429.19 & 12.83 & A91/683 & $46 X-2,119$ & $D$, alt. & 2.57 & -1.02 & 434.39 & 12.87 & A90/4545 & $46 \mathrm{X}-6,39$ & D. alt. & 1.75 & -0.87 \\
\hline 429.39 & 12.83 & A90/4838 & $46 X-2,139$ & G. venez & 1.97 & -0.52 & 434.59 & 12.87 & A91/3193 & $46 \mathrm{X}-6,59$ & Bulk & 1.63 & 0.25 \\
\hline 429.39 & 12.83 & A90/2995 & $46 \mathrm{X}-2,139$ & Bulk & 1.71 & 0.37 & 434.59 & 12.87 & A91/1741 & $46 X-6,59$ & D. alt. & 1.97 & -0.90 \\
\hline 429.59 & 12.83 & A90/2996 & $46 \mathrm{X}-3,9$ & Bulk & 1.68 & 0.40 & 434.59 & 12.87 & A91/1742 & $46 \mathrm{X}-6,59$ & G. venez. & 1.90 & -0.74 \\
\hline 429.59 & 12.83 & A90/4833 & $46 \mathrm{X}-3,9$ & G. venez. & 2.21 & -0.96 & 434.79 & 12.87 & A91/3194 & $46 \times-6,79$ & Bulk & 1.70 & 0.41 \\
\hline 429.59 & 12.83 & A90/4832 & $46 \mathrm{X}-3,9$ & D. alt. & 2.03 & -1.10 & 434.79 & 12.87 & A90/4535 & $46 X-6,79$ & D. alt. & 1.97 & -0.75 \\
\hline 429.79 & 12.83 & A90/4828 & $46 \mathrm{X}-3,29$ & G. venez. & 1.89 & -0.81 & 434.79 & 12.87 & A90/4536 & $46 \times-6,79$ & G. venez & 2.09 & -0.95 \\
\hline 429.79 & 12.83 & A91/1282 & $46 \mathrm{X}-3,29$ & Cib. mun. & 0.90 & 1.84 & 434.99 & 12.87 & A90/4532 & $46 \mathrm{X}-6,99$ & G. venez. & 2.03 & -0.65 \\
\hline 429.79 & 12.83 & A90/4827 & $46 \mathrm{X}-3,29$ & D. alt. & 2.00 & -0.98 & 434.99 & 12.87 & A91/3195 & $46 X-6,99$ & Bulk & 1.79 & 0.36 \\
\hline 429.79 & 12.83 & A90/2997 & $46 X-3,29$ & Bulk & 1.51 & 0.36 & 434.99 & 12.87 & A90/4531 & $46 \mathrm{X}-6,99$ & D. alt. & 2.12 & -0.92 \\
\hline 429.99 & 12.84 & A90/4726 & $46 \times-3,49$ & G. venez. & 2.23 & -0.99 & 435.19 & 12.88 & A90/4528 & $46 X-6,119$ & G. venez. & 1.64 & -0.48 \\
\hline 429.99 & 12.84 & A90/2998 & $46 \mathrm{X}-3,49$ & Bulk & 1.58 & 0.29 & 435.19 & 12.88 & A91/3196 & $46 X-6,119$ & Bulk & 1.80 & 0.33 \\
\hline 429.99 & 12.84 & A90/4725 & $46 \mathrm{X}-3,49$ & D. alt. & 2.15 & -0.97 & 435.19 & 12.88 & A90/4527 & $46 X-6,119$ & D. alt. & 2.22 & -0.96 \\
\hline 430.19 & 12.84 & A91/1747 & $46 X-3,69$ & D. alt. & 2.09 & -1.11 & 435.39 & 12.88 & A91/1046 & $46 X-6,139$ & D. alt. & 2.07 & -0.98 \\
\hline 430.19 & 12.84 & A91/1748 & $46 X-3,69$ & G. venez & 1.99 & -0.63 & 435.39 & 12.88 & A91/1047 & $46 X-6,139$ & G. venez. & 1.82 & -0.52 \\
\hline 430.19 & 12.84 & A90/2999 & $46 \mathrm{X}-3,69$ & Bulk & 1.62 & 0.27 & 435.39 & 12.88 & A91/3197 & $46 X-6,139$ & Bulk & 1.77 & 0.34 \\
\hline 430.39 & 12.84 & A90/4716 & $46 X-3,89$ & G. venez. & 1.76 & -0.83 & 435.59 & 12.88 & A91/1738 & $46 \times-7,9$ & G. venez. & 1.98 & -0.66 \\
\hline 430.39 & 12.84 & A $90 / 3000$ & $46 X-3,89$ & Bulk & 1.51 & 0.12 & 435.59 & 12.88 & A91/1737 & $46 X-7,9$ & D. alt. & 2.23 & -0.89 \\
\hline 430.39 & 12.84 & A90/4715 & $46 X-3,89$ & D. alt. & 2.07 & -0.97 & 435.59 & 12.88 & A91/1424 & $46 X-7,9$ & Cib. spp. & 1.18 & 2.16 \\
\hline 430.59 & 12.84 & A91/1289 & $46 X-3,109$ & Cib. mun. & 0.94 & 2.22 & 436.18 & 12.88 & A90/3018 & $47 X-1,8$ & Bulk & 1.66 & 0.02 \\
\hline 430.59 & 12.84 & A90/4710 & $46 X-3,109$ & D. alt. & 1.89 & -0.88 & 436.39 & 12.89 & A90/3019 & $47 X-1,29$ & Bulk & 1.63 & -0.04 \\
\hline 430.59 & 12.84 & A90/4711 & $46 X-3,109$ & G. venez. & 1.93 & -0.72 & 436.59 & 12.89 & A90/3020 & $47 X-1,49$ & Bulk & 1.68 & -0.01 \\
\hline 430.59 & 12.84 & A90/3001 & $46 \mathrm{X}-3,109$ & Bulk & 1.61 & 0.35 & 436.79 & 12.89 & A91/1423 & $47 X-1,69$ & Cib. spp. & 1.13 & 2.22 \\
\hline 430.79 & 12.84 & A90/3002 & $46 X-3,129$ & Bulk & 1.63 & 0.31 & 436.79 & 12.89 & A91/1422 & $47 X-1,69$ & $P$ wuell. & 1.18 & 2.08 \\
\hline 430.79 & 12.84 & A90/4705 & $46 X-3,129$ & D. alt. & 2.00 & -0.91 & 436.79 & 12.89 & A91/1740 & $47 X-1,69$ & G. venez & 1.77 & -0.64 \\
\hline 430.79 & 12.84 & A90/4706 & $46 \mathrm{X}-3,129$ & G. venez & 1.73 & -0.84 & 436.79 & 12.89 & A91/2430 & $47 X-1,69$ & D. alt. & 2.12 & -0.90 \\
\hline 431.19 & 12.84 & A $90 / 4700$ & $46 \mathrm{X}-4,19$ & D. alt. & 2.06 & -0.95 & 436.99 & 12.89 & A90/3021 & $47 X-1,89$ & Bulk & 1.77 & 0.02 \\
\hline 431.19 & 12.84 & A90/4701 & $46 \times-4,19$ & G. venez. & 1.85 & -1.12 & 437.19 & 12.89 & A90/3022 & $47 X-1,109$ & Bulk & 1.67 & 0.04 \\
\hline 431.19 & 12.84 & A91/3176 & $46 \times-4,19$ & Bulk & 1.60 & 0.19 & 437.39 & 12.89 & A90/3023 & $47 X-1,129$ & Bulk & 1.55 & 0.28 \\
\hline 431.39 & 12.85 & A90/4696 & $46 X-4,39$ & G. venez & 1.88 & -1.12 & 437.58 & 12.89 & A90/3024 & $47 X-1,148$ & Bulk & 1.58 & -0.02 \\
\hline 431.39 & 12.85 & A91/1286 & $46 X-4,39$ & Cib. mun. & 1.01 & 2.14 & 437.79 & 12.90 & A90/3025 & $47 X-2,19$ & Bulk & 1.52 & -0.01 \\
\hline 431.39 & 12.85 & A91/3177 & $46 X-4,36$ & Bulk & 1.61 & 0.32 & 437.99 & 12.90 & A90/3026 & $47 X-2,39$ & Bulk & 1.59 & -0.07 \\
\hline 431.59 & 12.85 & A91/3178 & $46 X-4,59$ & Bulk & 1.61 & 0.38 & 438.19 & 12.90 & A91/1420 & $47 X-2,59$ & P. wuell. & 0.89 & 2.13 \\
\hline 431.59 & 12.85 & A91/1745 & $46 X-4,59$ & D. alt. & 2.29 & -0.97 & 438.19 & 12.90 & A91/1733 & $47 X-2,59$ & D. alt. & 1.86 & -0.88 \\
\hline 431.59 & 12.85 & A91/1746 & $46 X-4,59$ & G. venez & 2.01 & -0.90 & 438.19 & 12.90 & A91/1421 & $47 X-2,59$ & Cib. spp. & 0.23 & 2.62 \\
\hline 431.79 & 12.85 & A90/4685 & $46 X-4,79$ & D. alt. & 2.21 & -1.10 & 438.19 & 12.90 & A91/1734 & $47 X-2,59$ & G. venez & 1.61 & -0.55 \\
\hline 431.79 & 12.85 & A91/3179 & $46 X-4,79$ & Bulk & 1.55 & 0.24 & 438.39 & 12.90 & A90/3027 & $47 X-2,79$ & Bulk & 1.51 & -0.16 \\
\hline 431.79 & 12.85 & A90/4686 & $46 X-4,79$ & G. venez & 2.00 & -1.06 & 438.59 & 12.90 & A90/3028 & $47 X-2,99$ & Bulk & 1.60 & -0.17 \\
\hline
\end{tabular}


Appendix B (continued).

\begin{tabular}{|c|c|c|c|c|c|c|c|c|c|c|c|c|c|}
\hline $\begin{array}{l}\text { Depth } \\
\text { (mbsf) }\end{array}$ & $\begin{array}{l}\text { Age } \\
(\mathrm{Ma})\end{array}$ & Lab no. & $\begin{array}{l}\text { Core, section, } \\
\text { interval }(\mathrm{cm})\end{array}$ & Species & $\delta^{13} \mathrm{C}$ & $\delta^{18} \mathrm{O}$ & $\begin{array}{l}\text { Depth } \\
\text { (mbsf) }\end{array}$ & $\begin{array}{l}\text { Age } \\
(\mathrm{Ma})\end{array}$ & Lab no. & $\begin{array}{l}\text { Core, section, } \\
\text { interval }(\mathrm{cm})\end{array}$ & Species & $\delta^{13} \mathrm{C}$ & $\delta^{18} \mathrm{O}$ \\
\hline 438.79 & 12.90 & A90/3029 & $47 X-2,119$ & Bulk & 1.59 & 0.11 & 454.49 & 13.03 & A90/3082 & $48 X-6,119$ & Bulk & 1.92 & -0.44 \\
\hline 438.99 & 12.91 & A $90 / 3030$ & $47 X-2,139$ & Bulk & 1.47 & -0.09 & 454.69 & 13.03 & A $90 / 3083$ & $48 X-6,139$ & Bulk & 1.72 & 0.19 \\
\hline 439.19 & 12.91 & A $90 / 3031$ & $47 X-3,9$ & Bulk & 1.54 & -0.07 & 454.89 & 13.03 & A $90 / 3084$ & $48 X-7,9$ & Bulk & 1.80 & -0.08 \\
\hline 439.39 & 12.91 & A $90 / 3032$ & $47 X-3,29$ & Bulk & 1.66 & 0.10 & 455.59 & 13.04 & A90/3085 & $49 X-1,9$ & Bulk & 1.79 & -0.12 \\
\hline 439.59 & 12.91 & A $90 / 3033$ & $47 X-3,49$ & Bulk & 1.48 & -0.12 & 455.79 & 13.04 & A90/3086 & $49 X-1,29$ & Bulk & 1.69 & -0.42 \\
\hline 439.79 & 12.91 & A91/1735 & $47 X-3,69$ & D. alt. & 2.05 & -0.95 & 455.99 & 13.04 & A90/3087 & $49 X-1,49$ & Bulk & 1.77 & -0.13 \\
\hline 439.79 & 12.91 & A91/1736 & $47 X-3,69$ & G. venez & 1.80 & -0.51 & 456.19 & 13.04 & A91/948 & $49 X-1,69$ & D. alt. & 2.11 & -1.14 \\
\hline 439.99 & 12.91 & A90/3034 & $47 X-3,89$ & Bulk & 1.55 & -0.29 & 456.19 & 13.04 & A91/949 & $49 X-1,69$ & G. venez & 1.73 & -0.67 \\
\hline 440.19 & 12.92 & A90/3035 & $47 X-3,109$ & Bulk & 1.47 & -0.13 & 456.19 & 13.04 & A91/1306 & $49 X-1,69$ & Cib. spp. & 1.76 & -1.10 \\
\hline 440.39 & 12.92 & A90/3036 & $47 X-3,129$ & Bulk & 1.38 & -0.03 & 456.39 & 13.04 & A90/3088 & $49 X-1,89$ & Bulk & 1.78 & -0.21 \\
\hline 440.58 & 12.92 & A90/3037 & $47 X-3,148$ & Bulk & 1.42 & 0.02 & 457.59 & 13.05 & A91/947 & $49 X-2,59$ & G. venez. & 1.54 & -0.68 \\
\hline 440.79 & 12.92 & A90/3038 & $47 X-4,19$ & Bulk & 1.28 & -0.01 & 457.59 & 13.05 & A91/946 & $49 X-2,59$ & D. alt. & 2.24 & -1.14 \\
\hline 440.99 & 12.92 & A90/3039 & $47 X-4,39$ & Bulk & 1.22 & 0.02 & 457.79 & 13.05 & A90/3089 & $49 X-2,79$ & Bulk & 1.86 & -0.55 \\
\hline 441.19 & 12.92 & A91/1313 & $47 X-4,59$ & $P$ wuell. & 0.71 & 2.05 & 458.99 & 13.06 & A $90 / 3090$ & $49 X-3,49$ & Bulk & 1.97 & -0.17 \\
\hline 441.19 & 12.92 & A91/1730 & $47 X-4,59$ & G. venez. & 1.46 & -0.53 & 459.19 & 13.06 & A91/944 & $49 X-3,69$ & $D$, alt. & 2.41 & -0.97 \\
\hline 441.19 & 12.92 & A91/1729 & $47 X-4,59$ & D. alt. & 1.66 & -0.92 & 459.19 & 13.06 & A91/945 & $49 X-3,69$ & G. venez. & 2.06 & -1.02 \\
\hline 441.39 & 12.92 & A90/3040 & $47 \times-4,79$ & Bulk & 1.22 & 0.04 & 460.59 & 13.08 & A91/943 & $49 X-4,59$ & G. venez & 2.14 & -0.98 \\
\hline 441.59 & 12.93 & A90/3041 & $47 X-4,99$ & Bulk & 1.28 & 0.18 & 460.59 & 13.08 & A91/1294 & $49 X-4,59$ & Cib. mun. & 1.15 & 1.74 \\
\hline 441.79 & 12.93 & A90/3042 & $47 X-4,119$ & Bulk & 1.34 & -0.09 & 460.59 & 13.08 & A91/942 & $49 X-4,59$ & D. alt. & 2.55 & -0.97 \\
\hline 441.99 & 12.93 & A90/3043 & $47 X-4,139$ & Bulk & 1.59 & -0.27 & 460.59 & 13.08 & A91/1295 & $49 X-4,59$ & $P$ wuell. & 1.24 & 1.58 \\
\hline 442.19 & 12.93 & A90/3044 & $47 X-5,9$ & Bulk & 1.48 & -0.08 & 460.79 & 13.08 & A90/3091 & $49 X-4,79$ & Bulk & 1.95 & -0.30 \\
\hline 442.39 & 12.93 & A90/3045 & $47 X-5,29$ & Bulk & 1.40 & -0.25 & 462.19 & 13.09 & A $91 / 940$ & $49 X-5,69$ & D. alt. & 2.43 & -0.88 \\
\hline 442.59 & 12.93 & $\mathrm{~A} 90 / 3046$ & $47 X-5,49$ & Bulk & 1.45 & 0.03 & 462.19 & 13.09 & A91/941 & $49 X-5,69$ & G. venez. & 1.84 & -0.54 \\
\hline 442.79 & 12.94 & A91/1731 & $47 X-5,69$ & D. alt. & 1.97 & -1.05 & 462.39 & 13.09 & A $90 / 3092$ & $49 X-5,89$ & Bulk & 1.84 & -0.13 \\
\hline 442.79 & 12.94 & A91/1311 & $47 X-5,69$ & P. wuell. & 1.06 & 2.03 & 463.59 & 13.10 & A91/938 & $49 X-6,59$ & D. alt. & 2.49 & -1.08 \\
\hline 442.79 & 12.94 & A91/1312 & $47 X-5,69$ & Cib. spp. & 0.99 & 1.63 & 463.59 & 13.10 & A91/939 & $49 X-6,59$ & G. venez & 2.01 & -0.51 \\
\hline 442.79 & 12.94 & A91/1732 & $47 X-5,69$ & G. venez. & 1.78 & -0.63 & 463.79 & 13.10 & A90/3093 & $49 X-6,79$ & Bulk & 1.81 & -0.31 \\
\hline 445.89 & 12.96 & A90/3047 & $48 X-1,9$ & Bulk & 1.47 & -0.30 & 464.29 & 13.13 & A90/3095 & $50 X-1,69$ & Bulk & 1.86 & -0.47 \\
\hline 446.09 & 12.96 & A90/3048 & $48 X-1,29$ & Bulk & 1.45 & -0.44 & 464.29 & 13.13 & A91/936 & $50 X-1,69$ & D. alt. & 2.13 & -1.05 \\
\hline 446.29 & 12.96 & A90/3049 & $48 X-1,49$ & Bulk & 1.71 & -0.31 & 464.29 & 13.13 & A91/937 & $50 X-1,69$ & G. venez. & 1.60 & -0.33 \\
\hline 446.49 & 12.96 & A91/1032 & $48 X-1,69$ & D. alt. & 2.25 & -1.00 & 464.79 & 13.16 & A90/3094 & $49 X-7,29$ & Bulk & 1.75 & -0.46 \\
\hline 446.49 & 12.96 & A91/1033 & $48 X-1,69$ & G. venez & 1.61 & -0.36 & 465.69 & 13.21 & A91/935 & $50 \times-2,59$ & G. venez. & 1.78 & 0.12 \\
\hline 446.69 & 12.97 & A90/3050 & $48 \mathrm{X}-1,89$ & Bulk & 1.72 & -0.34 & 465.69 & 13.21 & A91/934 & $50 \times-2,59$ & D. alt. & 2.24 & -1.06 \\
\hline 446.89 & 12.97 & A90/3051 & $48 X-1,109$ & Bulk & 1.65 & -0.21 & 465.69 & 13.21 & A $90 / 3096$ & $50 X-2,59$ & Bulk & 2.03 & -0.63 \\
\hline 447.09 & 12.97 & A90/3052 & $48 X-1,129$ & Bulk & 1.72 & -0.43 & 467.29 & 13.30 & A90/3097 & $50 \times-3,69$ & Bulk & 1.84 & -0.37 \\
\hline 447.28 & 12.97 & A90/3053 & $48 \mathrm{X}-1,148$ & Bulk & 1.71 & -0.58 & 467.29 & 13.30 & A91/811 & $50 X-3,69$ & G. venez. & 1.56 & -0.37 \\
\hline 447.49 & 12.97 & A90/3054 & $48 X-2,19$ & Bulk & 1.74 & -0.47 & 467.29 & 13.30 & A91/810 & $50 X-3,69$ & D. alt. & 2.06 & -1.25 \\
\hline 447.69 & 12.97 & A90/3055 & $48 X-2,39$ & Bulk & 1.77 & -0.51 & 468.69 & 13.37 & A91/808 & $50 \times-4,59$ & D. alt. & 2.13 & -0.96 \\
\hline 447.89 & 12.98 & A91/1031 & $48 X-2,59$ & G. venez & 1.88 & -0.70 & 468.69 & 13.37 & A $90 / 3098$ & $50 \times-4,59$ & Bulk & 2.00 & -0.56 \\
\hline 447.89 & 12.98 & A91/1030 & $48 X-2,59$ & D. alt. & 2.37 & -0.97 & 468.69 & 13.37 & A91/809 & $50 \times-4,59$ & G. venez. & 2.33 & -0.92 \\
\hline 448.09 & 12.98 & A90/3056 & $48 X-2,79$ & Bulk & 1.69 & -0.47 & 470.29 & 13.46 & A $90 / 3099$ & $50 X-5,69$ & Bulk & 2.02 & -0.64 \\
\hline 448.29 & 12.98 & A90/3057 & $48 X-2,99$ & Bulk & 1.61 & -0.36 & 470.29 & 13.46 & A91/1343 & $50 X-5,69$ & $P$ wuell. & 1.49 & 1.81 \\
\hline 448.49 & 12.98 & A90/3058 & $48 X-2,119$ & Bulk & 1.57 & -0.29 & 471.69 & 13.54 & A91/804 & $50 X-6,59$ & D. alt. & 2.35 & -1.02 \\
\hline 448.69 & 12.98 & A90/3059 & $48 X-2,139$ & Bulk & 1.47 & -0.28 & 471.69 & 13.54 & A91/805 & $50 \times-6,59$ & G. venez. & 1.69 & -0.54 \\
\hline 448.89 & 12.98 & A90/3060 & $48 X-3,9$ & Bulk & 1.51 & -0.15 & 471.69 & 13.54 & A91/1340 & $50 \times-6,59$ & $P$ wuell. & 1.27 & 1.94 \\
\hline 449.09 & 12.99 & A90/3061 & $48 X-3,39$ & Bulk & 1.53 & -0.30 & 471.69 & 13.54 & A $90 / 3100$ & $50 X-6,59$ & Bulk & 1.87 & -0.51 \\
\hline 449.29 & 12.99 & A90/3062 & $48 X-3,49$ & Bulk & 1.54 & -0.42 & 473.99 & 13.66 & A91/1342 & $51 X-1,69$ & Cib. spp. & 1.49 & 1.71 \\
\hline 449.49 & 12.99 & A91/1028 & $48 X-3,69$ & D. alt. & 2.04 & -1.13 & 473.99 & 13.66 & A91/803 & $51 X-1,69$ & G. venez. & 1.82 & -0.53 \\
\hline 449.49 & 12.99 & A91/1029 & $48 X-3,69$ & $G$. venez & 1.82 & -0.66 & 473.99 & 13.66 & A91/1341 & $51 X-1,69$ & $P$ wuell. & 1.97 & 1.99 \\
\hline 449.69 & 12.99 & A90/3063 & $48 X-3,89$ & Bulk & 1.50 & -0.43 & 473.99 & 13.66 & A90/3154 & $51 X-1,69$ & Bulk & 2.55 & -0.32 \\
\hline 449.89 & 12.99 & A90/3064 & $48 X-3,109$ & Bulk & 1.44 & -0.35 & 473.99 & 13.66 & A91/802 & $51 X-1,69$ & D. alt. & 2.93 & -0.92 \\
\hline 450.49 & 13.00 & A90/3065 & $48 X-4,19$ & Bulk & 1.54 & 0.27 & 475.39 & 13.72 & A91/1339 & $51 X-2,59$ & $P$ wuell. & 1.87 & 1.86 \\
\hline 450.69 & 13.00 & A90/3066 & $48 X-4,39$ & Bulk & 1.44 & -0.39 & 475.39 & 13.72 & A $90 / 3155$ & $51 X-2,59$ & Bulk & 2.40 & -0.38 \\
\hline 450.89 & 13.00 & A91/1026 & $48 X-4,59$ & D. alt. & 1.87 & -0.94 & 475.39 & 13.72 & A91/800 & $51 X-2,59$ & D. alt. & 2.96 & -0.89 \\
\hline 450.89 & 13.00 & A91/1027 & $48 X-4,59$ & G. venez & 1.66 & -0.75 & 475.39 & 13.72 & A91/801 & $51 X-2,59$ & G. venez. & 2.50 & -0.62 \\
\hline 451.09 & 13.00 & A90/3067 & $48 \times-4,79$ & Bulk & 1.48 & -0.48 & 476.99 & 13.80 & A91/1337 & $51 X-3,69$ & P. wuell. & 1.71 & 1.87 \\
\hline 451.29 & 13.00 & A90/3068 & $48 X-4,99$ & Bulk & 1.64 & -0.49 & 476.99 & 13.80 & A91/799 & $51 X-3,69$ & G. venez & 2.20 & -0.47 \\
\hline 451.49 & 13.00 & A90/3069 & $48 X-4,119$ & Bulk & 1.54 & -0.33 & 476.99 & 13.80 & A91/1338 & $51 X-3,69$ & Cib. spp. & 1.65 & 1.85 \\
\hline 451.69 & 13.01 & A90/3070 & $48 X-4,139$ & Bulk & 1.67 & -0.31 & 476.99 & 13.80 & A91/798 & $51 X-3,69$ & D. alt. & 2.98 & -1.06 \\
\hline 451.89 & 13.01 & A90/3071 & $48 X-5,9$ & Bulk & 1.79 & -0.20 & 476.99 & 13.80 & A $90 / 3156$ & $51 X-3,69$ & Bulk & 2.33 & -0.21 \\
\hline 452.09 & 13.01 & A90/3072 & $48 X-5,29$ & Bulk & 1.77 & -0.30 & 478.39 & 13.86 & A91/1336 & $51 \times-4,59$ & P. wuell. & 1.85 & 1.91 \\
\hline 452.29 & 13.01 & A90/3073 & $48 X-5,49$ & Bulk & 1.83 & -0.22 & 478.39 & 13.86 & A91/795 & $51 X-4,59$ & G. venez & 2.27 & -0.04 \\
\hline 452.49 & 13.01 & A91/1309 & $48 X-5,69$ & $P$ wuell. & 1.14 & 1.89 & 478.39 & 13.86 & A $90 / 3157$ & $51 X-4,59$ & Bulk & 2.72 & 0.20 \\
\hline 452.49 & 13.01 & A91/1310 & $48 X-5,69$ & Cib. spp. & 0.36 & 2.17 & 478.39 & 13.86 & A $91 / 794$ & $51 X-4,59$ & D. alt. & 2.85 & -1.13 \\
\hline 452.49 & 13.01 & A91/1025 & $48 X-5,69$ & G. venez & 1.74 & -0.60 & 479.99 & 13.94 & A91/793 & $51 X-5,69$ & G. venez & 2.65 & 0.09 \\
\hline 452.49 & 13.01 & A91/1024 & $48 X-5,69$ & D. alt. & 2.24 & -0.91 & 479.99 & 13.94 & A $90 / 3158$ & $51 X-5,69$ & Bulk & 2.30 & 0.35 \\
\hline 452.69 & 13.01 & A90/3074 & $48 X-5,89$ & Bulk & 1.83 & -0.33 & 479.99 & 13.94 & A91/1335 & $51 X-5,69$ & P. wuell. & 2.11 & 1.94 \\
\hline 452.89 & 13.02 & A90/3075 & $48 X-5,109$ & Bulk & 1.63 & -0.25 & 479.99 & 13.94 & A $91 / 792$ & $51 X-5,69$ & D. alt. & 2.87 & -0.62 \\
\hline 453.09 & 13.02 & A90/3076 & $48 X-5,129$ & Bulk & 1.70 & -0.32 & 481.39 & 14.00 & A $91 / 782$ & $51 X-6,59$ & D. alt. & 2.83 & -1.07 \\
\hline 453.28 & 13.02 & A90/3077 & $48 X-5,148$ & Bulk & 1.57 & -0.39 & 481.39 & 14.00 & A91/1305 & $51 X-6,59$ & P. wuell. & 1.85 & 1.82 \\
\hline 453.49 & 13.02 & A90/3078 & $48 X-6,19$ & Bulk & 1.53 & -0.27 & 481.39 & 14.00 & A $90 / 3713$ & $51 X-6,59$ & Bulk & 2.23 & 0.11 \\
\hline 453.69 & 13.02 & A90/3079 & $48 X-6,39$ & Bulk & 1.63 & -0.41 & 481.39 & 14.00 & A $91 / 783$ & $51 X-6,59$ & G. venez & 2.07 & 0.09 \\
\hline 453.89 & 13.02 & A91/1022 & $48 X-6,59$ & D. alt. & 2.07 & -1.05 & 482.59 & 14.06 & A91/933 & $51 X-7,29$ & G. venez & 2.23 & -0.01 \\
\hline 453.89 & 13.02 & A91/1308 & $48 X-6,59$ & Cib. spp. & 1.69 & -1.18 & 482.59 & 14.06 & A91/932 & $51 X-7,29$ & D. alt. & 2.65 & -0.90 \\
\hline 453.89 & 13.02 & A91/1023 & $48 X-6,59$ & G. venez & 1.69 & -0.78 & 482.59 & 14.06 & A $91 / 1304$ & $51 X-7,29$ & P. wuell. & 1.89 & 1.87 \\
\hline 453.89 & 13.02 & A91/1307 & $48 X-6,59$ & $P$ wuell. & 1.03 & 2.26 & 482.59 & 14.06 & A $90 / 3714$ & $51 X-7,29$ & Bulk & 2.30 & 0.35 \\
\hline 454.09 & 13.02 & A90/3080 & $48 X-6,79$ & Bulk & 1.73 & -0.20 & 483.29 & 14.09 & A $90 / 3715$ & $52 X-1,69$ & Bulk & 2.07 & 0.31 \\
\hline 454.29 & 13.03 & A $90 / 3081$ & $48 X-6,99$ & Bulk & 1.82 & -0.25 & 483.29 & 14.09 & A91/931 & $52 X-1,69$ & G. venez & 2.14 & -0.71 \\
\hline
\end{tabular}


Appendix B (continued).

\begin{tabular}{|c|c|c|c|c|c|c|c|c|c|c|c|c|c|}
\hline $\begin{array}{l}\text { Depth } \\
\text { (mbsf) }\end{array}$ & $\begin{array}{l}\text { Age } \\
(\mathrm{Ma})\end{array}$ & Lab no. & $\begin{array}{l}\text { Core, section, } \\
\text { interval }(\mathrm{cm})\end{array}$ & Species & $\delta^{13} \mathrm{C}$ & $\delta^{18} \mathrm{O}$ & $\begin{array}{l}\text { Depth } \\
\text { (mbsf) }\end{array}$ & $\begin{array}{l}\text { Age } \\
\text { (Ma) }\end{array}$ & Lab no. & $\begin{array}{l}\text { Core, section, } \\
\text { interval }(\mathrm{cm})\end{array}$ & Species & $\delta^{13} \mathrm{C}$ & $\delta^{18} \mathrm{O}$ \\
\hline 483.29 & 14.09 & A91/1300 & $52 X-1,69$ & P. wuell. & 1.34 & 1.57 & 515.29 & 15.60 & A91/796 & $55 X-3,69$ & D. alt. & 2.89 & -1.23 \\
\hline 483.29 & 14.09 & A91/930 & $52 X-1,69$ & D. alt. & 2.35 & -1.08 & 515.29 & 15.60 & A91/1293 & $55 X-3,69$ & P. wuell. & 1.51 & 1.21 \\
\hline 484.69 & 14.16 & A91/1299 & $52 X-2,59$ & Cib. spp. & 1.12 & 1.28 & 516.69 & 15.66 & A91/788 & $55 X-4,59$ & D. alt. & 2.80 & -1.29 \\
\hline 484.69 & 14.16 & A91/928 & $52 X-2,59$ & D. alt. & 2.17 & -1.09 & 516.69 & 15.66 & A91/1297 & $55 X-4,59$ & Cib. mun. & 1.26 & 1.11 \\
\hline 484.69 & 14.16 & A90/3716 & $52 X-2,59$ & Bulk & 1.89 & -0.01 & 516.69 & 15.66 & A90/3752 & $55 X-4,59$ & Bulk & 2.11 & -0.09 \\
\hline 484.69 & 14.16 & A91/929 & $52 X-2,59$ & G. venez. & 1.96 & -0.79 & 516.69 & 15.66 & A91/789 & $55 \times-4,59$ & G. venez. & 2.00 & -0.50 \\
\hline 484.69 & 14.16 & A91/1298 & $52 X-2,59$ & P. wuell. & 1.25 & 1.09 & 516.69 & 15.66 & A91/1296 & $55 X-4,59$ & P. wuell. & 1.52 & 1.16 \\
\hline 486.29 & 14.23 & A91/1334 & $52 X-3,69$ & Cib. spp. & 1.40 & 1.29 & 518.29 & 15.74 & A91/786 & $55 X-5,69$ & D. alt. & 2.39 & -1.57 \\
\hline 486.29 & 14.23 & A91/926 & $52 X-3,69$ & D. alt. & 2.44 & -1.21 & 518.29 & 15.74 & A91/1283 & $55 X-5,69$ & Cib. mun. & 1.26 & 0.90 \\
\hline 486.29 & 14.23 & A91/1333 & $52 \times-3,69$ & $P$ wuell. & 1.45 & 1.24 & 518.29 & 15.74 & A90/3753 & $55 X-5,69$ & Bulk & 1.98 & -0.24 \\
\hline 486.29 & 14.23 & A91/927 & $52 X-3,69$ & G. venez & 1.92 & -0.70 & 518.29 & 15.74 & A91/807 & $55 X-5,69$ & G. venez & 2.05 & -0.42 \\
\hline 486.29 & 14.23 & A90/3735 & $52 X-3,69$ & Bulk & 1.94 & -0.44 & 519.69 & 15.81 & A90/3754 & $55 \times-6,59$ & Bulk & 2.08 & -0.12 \\
\hline 487.69 & 14.30 & A90/3736 & $52 \times-4,59$ & Bulk & 1.83 & -0.17 & 519.69 & 15.81 & A91/3198 & $55 X-6,59$ & Cib. spp. & 2.21 & 0.01 \\
\hline 487.69 & 14.30 & A91/924 & $52 X-4,59$ & D. alt. & 2.30 & -0.65 & 519.69 & 15.81 & A91/784 & $55 X-6,59$ & D. alt. & 2.78 & -1.04 \\
\hline 487.69 & 14.30 & A91/925 & $52 X-4,59$ & G. venez & 1.93 & -0.37 & 519.69 & 15.81 & A91/785 & $55 X-6,59$ & G. venez. & 2.13 & 0.03 \\
\hline 487.69 & 14.30 & A91/1332 & $52 X-4,59$ & P. wuell. & 1.44 & 1.48 & 520.89 & 15.86 & A90/3755 & $55 X-7,29$ & Bulk & 1.96 & -0.36 \\
\hline 489.29 & 14.38 & A91/922 & $52 X-5,69$ & D. alt. & 2.55 & -0.90 & 520.89 & 15.86 & A91/3199 & $55 X-7,29$ & Cib. spp. & 1.40 & 1.64 \\
\hline 489.29 & 14.38 & A90/3737 & $52 X-5,69$ & Bulk & 2.06 & -0.36 & 521.99 & 15.91 & A91/697 & $56 \mathrm{X}-1,69$ & G. venez & 2.25 & -0.25 \\
\hline 489.29 & 14.38 & A91/923 & $52 X-5,69$ & G. venez & 2.10 & -0.31 & 521.99 & 15.91 & A90/3756 & $56 X-1,69$ & Bulk & 2.23 & -0.26 \\
\hline 489.29 & 14.38 & A91/1331 & $52 X-5,69$ & Cib. spp. & 1.74 & 1.65 & 521.99 & 15.91 & A91/3200 & $56 \mathrm{X}-1,69$ & Cib. spp. & 2.02 & 1.34 \\
\hline 489.29 & 14.38 & A91/1330 & $52 X-5,69$ & P. wuell. & 1.67 & 1.39 & 521.99 & 15.91 & A91/696 & $56 \mathrm{X}-1,69$ & D. alt. & 2.69 & -1.10 \\
\hline 492.99 & 14.55 & A91/1329 & $53 X-1,69$ & P. wuell. & 1.66 & 1.57 & 523.39 & 15.98 & A90/3757 & $56 \mathrm{X}-2,59$ & Bulk & 2.07 & -0.13 \\
\hline 492.99 & 14.55 & A90/3738 & $53 X-1,69$ & Bulk & 2.07 & -0.12 & 523.39 & 15.98 & A91/695 & $56 X-2,59$ & G. venez. & 2.15 & -0.33 \\
\hline 492.99 & 14.55 & A91/920 & $53 X-1,69$ & D. alt. & 2.25 & -1.02 & 523.39 & 15.98 & A91/694 & $56 X-2,59$ & D. alt. & 2.50 & -1.00 \\
\hline 492.99 & 14.55 & A91/921 & $53 \mathrm{X}-1,69$ & G. venez. & 2.09 & -0.27 & 523.39 & 15.98 & A91/3201 & $56 X-2,59$ & Cib. spp. & 2.11 & -0.58 \\
\hline 494.39 & 14.62 & A91/919 & $53 X-2,59$ & G. venez & 2.32 & -0.32 & 524.99 & 16.06 & A90/3758 & $56 X-3,69$ & Bulk & 2.28 & 0.00 \\
\hline 494.39 & 14.62 & A91/918 & $53 X-2,59$ & D. alt. & 2.60 & -1.17 & 524.99 & 16.06 & A91/3202 & $56 X-3,69$ & $G$-cass. spp. & 1.38 & 1.97 \\
\hline 494.39 & 14.62 & A91/1327 & $53 X-2,59$ & P. wuell. & 2.01 & 1.34 & 526.39 & 16.12 & A $91 / 690$ & $56 X-4,59$ & D. alt. & 2.15 & -1.56 \\
\hline 494.39 & 14.62 & A91/1328 & $53 X-2,59$ & Cib. spp. & 1.82 & 1.45 & 526.39 & 16.12 & A91/3204 & $56 X-4,59$ & Cib. spp. & 0.82 & 1.20 \\
\hline 494.39 & 14.62 & A90/3739 & $53 X-2,59$ & Bulk & 2.29 & 0.03 & 526.39 & 16.12 & A91/691 & $56 \times-4,59$ & G. venez & 1.92 & -0.62 \\
\hline 495.99 & 14.69 & A91/1325 & $53 X-3,69$ & P. wuell. & 1.84 & 1.25 & 526.39 & 16.12 & A90/3759 & $56 \times-4,59$ & Bulk & 1.83 & 0.05 \\
\hline 495.99 & 14.69 & A91/1326 & $53 \mathrm{X}-3,69$ & Cib. spp. & 1.34 & 1.90 & 531.59 & 16.37 & A90/3760 & $57 X-1,69$ & Bulk & 2.22 & -0.31 \\
\hline 495.99 & 14.69 & A90/3740 & $53 X-3,69$ & Bulk & 2.27 & -0.13 & 531.59 & 16.37 & A91/689 & $57 X-1,69$ & G. venez. & 2.29 & -0.59 \\
\hline 495.99 & 14.69 & A91/916 & $53 X-3,69$ & D. alt. & 2.47 & -1.00 & 531.59 & 16.37 & A91/688 & $57 X-1,69$ & D. alt. & 2.59 & -1.23 \\
\hline 495.99 & 14.69 & A91/917 & $53 \times-3,69$ & G. venez. & 2.23 & -0.40 & 531.59 & 16.37 & A91/3205 & $57 X-1,69$ & Cib. spp. & 0.92 & 1.44 \\
\hline 497.39 & 14.76 & A91/915 & $53 X-4,59$ & G. venez. & 2.07 & 0.01 & 532.99 & 16.43 & A91/685 & $57 X-2,59$ & D. alt. & 2.78 & -1.06 \\
\hline 497.39 & 14.76 & A91/1316 & $53 X-4,59$ & Cib. spp. & 1.81 & 1.43 & 532.99 & 16.43 & A91/3207 & $57 X-2,59$ & Cib. spp. & 0.99 & 1.91 \\
\hline 497.39 & 14.76 & A91/914 & $53 X-4,59$ & D. alt. & 2.28 & -0.87 & 532.99 & 16.43 & A90/3761 & $57 X-2,59$ & Bulk & 2.07 & -0.19 \\
\hline 497.39 & 14.76 & A90/3741 & $53 X-4,59$ & Bulk & 2.25 & -0.03 & 532.99 & 16.43 & A91/686 & $57 X-2,59$ & G. venez & 2.22 & 0.00 \\
\hline 502.59 & 15.00 & A91/1324 & $54 X-1,69$ & $P$. wuell. & 1.86 & 1.59 & 534.59 & 16.51 & A91/822 & $57 X-3,69$ & D. alt. & 2.61 & -1.32 \\
\hline 502.59 & 15.00 & A91/841 & $54 X-1,69$ & G. venez. & 2.43 & -0.51 & 534.59 & 16.51 & A91/823 & $57 X-3,69$ & G. venez & 2.44 & -0.87 \\
\hline 502.59 & 15.00 & A90/3742 & $54 X-1,69$ & Bulk & 2.31 & 0.08 & 534.59 & 16.51 & A90/3762 & $57 X-3,69$ & Bulk & 2.09 & -0.33 \\
\hline 502.59 & 15.00 & A91/840 & $54 X-1,69$ & D. alt. & 2.72 & -0.98 & 535.99 & 16.57 & A91/821 & $57 X-4,59$ & $G$, venez & 2.51 & -0.64 \\
\hline 503.79 & 15.06 & A90/3743 & $54 X-2,59$ & Bulk & 2.15 & -0.02 & 535.99 & 16.57 & A90/3763 & $57 X-4,59$ & Bulk & 2.12 & -0.23 \\
\hline 503.99 & 15.07 & A91/839 & $54 X-2,59$ & G. venez. & 2.00 & -0.12 & 535.99 & 16.57 & A91/820 & $57 X-4,59$ & $D$, alt. & 2.67 & -0.97 \\
\hline 503.99 & 15.07 & A91/838 & $54 X-2,59$ & D. alt. & 2.36 & -1.20 & 535.99 & 16.57 & A91/3208 & $57 X-4,59$ & Cib. spp. & 1.57 & 1.62 \\
\hline 503.99 & 15.07 & A91/1323 & $54 X-2,59$ & $P$ wuell. & 1.71 & 1.53 & 537.59 & 16.65 & A91/818 & $57 X-5,69$ & D. alt. & 2.58 & -0.99 \\
\hline 505.59 & 15.14 & A91/836 & $54 X-3,69$ & D. alt. & 2.37 & -1.15 & 537.59 & 16.65 & A91/819 & $57 X-5,69$ & G. venez & 2.31 & -0.06 \\
\hline 505.59 & 15.14 & A91/837 & $54 X-3,69$ & G. venez. & 2.01 & -0.59 & 537.59 & 16.65 & A90/3764 & $57 X-5,69$ & Bulk & 1.88 & -0.16 \\
\hline 505.59 & 15.14 & A91/1322 & $54 X-3,69$ & $P$ wuell. & 1.10 & 1.37 & 537.59 & 16.65 & A91/3209 & $57 X-5,69$ & Cib. spp. & 0.88 & 0.91 \\
\hline 505.59 & 15.14 & A90/3744 & $54 X-3,69$ & Bulk & 2.21 & -0.06 & 538.99 & 16.71 & A90/3765 & $57 X-6,59$ & Bulk & 1.82 & -0.22 \\
\hline 506.99 & 15.21 & A91/835 & $54 X-4,59$ & G. venez. & 2.03 & -0.25 & 538.99 & 16.71 & A91/3210 & $57 X-6,59$ & Cib. spp. & 1.32 & 1.36 \\
\hline 506.99 & 15.21 & A90/3745 & $54 X-4,59$ & Bulk & 2.36 & -0.04 & 538.99 & 16.71 & A91/817 & $57 X-6,59$ & G. venez & 2.03 & -0.80 \\
\hline 506.99 & 15.21 & A91/834 & $54 X-4,59$ & D. alt. & 2.57 & -1.07 & 538.99 & 16.71 & A91/816 & $57 X-6,59$ & D. alt. & 2.51 & -0.88 \\
\hline 506.99 & 15.21 & A91/1320 & $54 X-4,59$ & P. wuell. & 1.58 & 1.03 & 540.19 & 16.77 & A91/3212 & $57 X-7,29$ & Cib. spp. & 1.75 & 0.88 \\
\hline 508.59 & 15.28 & A91/833 & $54 X-5,69$ & G. venez. & 1.90 & -0.31 & 540.19 & 16.77 & A91/815 & $57 X-7,29$ & G. venez. & 2.35 & -0.25 \\
\hline 508.59 & 15.28 & A91/832 & $54 X-5,69$ & D. alt. & 2.18 & -1.01 & 540.19 & 16.77 & A91/814 & $57 X-7,29$ & D. alt. & 2.55 & -0.90 \\
\hline 508.59 & 15.28 & A90/3746 & $54 X-5,69$ & Bulk & 2.05 & 0.12 & 540.19 & 16.77 & A90/3766 & $57 X-7,29$ & Bulk & 1.99 & -0.13 \\
\hline 509.99 & 15.35 & A91/1317 & $54 X-6,59$ & $P$. wuell. & 1.65 & 1.16 & 541.19 & 16.82 & A90/3767 & $58 X-1,69$ & Bulk & 2.16 & -0.06 \\
\hline 509.99 & 15.35 & A91/1318 & $54 X-6,59$ & Cib. spp. & 1.80 & 0.25 & 541.19 & 16.82 & A91/813 & $58 X-1,69$ & G. venez. & 2.09 & -1.07 \\
\hline 509.99 & 15.35 & A91/831 & $54 X-6,59$ & G. venez & 2.07 & -0.56 & 541.19 & 16.82 & A91/3213 & $58 \mathrm{X}-1,69$ & Cib. spp. & 1.18 & 1.38 \\
\hline 509.99 & 15.35 & A90/3747 & $54 X-6,59$ & Bulk & 2.13 & -0.17 & 541.19 & 16.82 & A91/812 & $58 X-1,69$ & D. alt. & 2.63 & -0.94 \\
\hline 509.99 & 15.35 & A91/830 & $54 X-6,59$ & D. alt. & 2.38 & -1.13 & 542.59 & 16.88 & A91/3214 & $58 X-2,59$ & Cib. spp. & 2.01 & 1.32 \\
\hline 511.19 & 15.41 & A90/3748 & $54 X-7,29$ & Bulk & 2.41 & -0.09 & 542.59 & 16.88 & A91/791 & $58 X-2,59$ & G. venez & 2.38 & -0.36 \\
\hline 511.19 & 15.41 & A91/829 & $54 X-7,29$ & $G$, venez & 2.24 & -0.37 & 542.59 & 16.88 & A90/3768 & $58 X-2,59$ & Bulk & 2.19 & 0.00 \\
\hline 511.19 & 15.41 & A91/828 & $54 X-7,29$ & D. alt. & 2.67 & -1.03 & 542.59 & 16.88 & A91/790 & $58 X-2,59$ & D. alt. & 3.11 & -0.98 \\
\hline 511.89 & 15.44 & A91/699 & $55 \mathrm{X}-1,29$ & $G$. venez. & 2.05 & -0.50 & 544.19 & 16.96 & A91/781 & $58 \mathrm{X}-3,69$ & G. venez. & 1.88 & -0.74 \\
\hline 511.89 & 15.44 & A91/698 & $55 \mathrm{X}-1,29$ & D. alt. & 2.39 & -1.16 & 544.19 & 16.96 & A91/3216 & $58 X-3,69$ & Cib. spp. & 1.04 & 1.49 \\
\hline 512.29 & 15.46 & A91/1302 & $55 X-1,69$ & P. wuell. & 1.75 & 1.01 & 544.19 & 16.96 & A90/3769 & $58 X-3,69$ & Bulk & 1.87 & -0.23 \\
\hline 512.29 & 15.46 & A91/827 & $55 X-1,69$ & G. venez. & 2.44 & -0.75 & 544.19 & 16.96 & A91/780 & $58 X-3,69$ & D. alt. & 2.42 & -1.14 \\
\hline 512.29 & 15.46 & A91/826 & $55 \mathrm{X}-1,69$ & D. alt. & 2.66 & -1.23 & 545.59 & 17.03 & A91/779 & $58 X-4,59$ & G. venez & 2.17 & -0.90 \\
\hline 512.29 & 15.46 & A90/3749 & $55 \mathrm{X}-1,69$ & Bulk & 2.35 & 0.14 & 545.59 & 17.03 & A90/3709 & $58 X-4,59$ & Bulk & 2.14 & 0.07 \\
\hline 512.29 & 15.46 & A91/1301 & $55 X-1,69$ & Cib. spp. & 1.29 & 1.17 & 545.79 & 17.03 & A91/778 & $58 X-4,59$ & D. alt. & 2.50 & -0.99 \\
\hline 513.69 & 15.52 & A91/824 & $55 \mathrm{X}-2,59$ & D. alt. & 2.85 & -1.12 & 547.19 & 17.10 & A91/777 & $58 X-5,69$ & G. venez & 2.08 & -0.99 \\
\hline 513.69 & 15.52 & A90/3750 & $55 X-2,59$ & Bulk & 2.16 & -0.15 & 547.19 & 17.10 & A91/3218 & $58 X-5,69$ & Cib. spp. & 1.54 & 1.16 \\
\hline 513.69 & 15.52 & A91/825 & $55 \mathrm{X}-2,59$ & G. venez & 2.37 & -0.86 & 547.19 & 17.10 & A91/776 & $58 X-5,69$ & D. alt. & 2.74 & -1.21 \\
\hline 515.29 & 15.60 & A91/693 & $55 X-3,69$ & $G$. venez & 2.03 & -0.30 & 547.19 & 17.10 & A90/3710 & $58 X-5,69$ & Bulk & 1.79 & -0.20 \\
\hline 515.29 & 15.60 & A90/3751 & $55 X-3,69$ & Bulk & 2.32 & 0.04 & 548.59 & 17.17 & A91/775 & $58 X-6,59$ & G. venez & 1.93 & -0.35 \\
\hline
\end{tabular}


Appendix B (continued).

\begin{tabular}{|c|c|c|c|c|c|c|c|c|c|c|c|c|c|}
\hline $\begin{array}{l}\text { Depth } \\
\text { (mbsf) }\end{array}$ & $\begin{array}{l}\text { Age } \\
(\mathrm{Ma})\end{array}$ & Lab no. & $\begin{array}{l}\text { Core, section, } \\
\text { interval }(\mathrm{cm})\end{array}$ & Species & $\delta^{13} \mathrm{C}$ & $\delta^{18} \mathrm{O}$ & $\begin{array}{l}\text { Depth } \\
\text { (mbsf) }\end{array}$ & $\begin{array}{l}\text { Age } \\
(\mathrm{Ma})\end{array}$ & Lab no. & $\begin{array}{l}\text { Core, section, } \\
\text { interval }(\mathrm{cm})\end{array}$ & Species & $\delta^{13} \mathrm{C}$ & $\delta^{18} \mathrm{O}$ \\
\hline 548.59 & 17.17 & A91/774 & $58 X-6,59$ & D. alt. & 2.21 & -1.06 & 576.29 & 18.47 & A90/3117 & $61 X-5,69$ & Bulk & 1.23 & -0.29 \\
\hline 548.59 & 17.17 & A $90 / 3772$ & $58 X-6,59$ & Bulk & 1.91 & -0.23 & 576.29 & 18.47 & A91/2790 & $61 X-5,69$ & D. alt. & 2.18 & -0.96 \\
\hline 549.79 & 17.22 & A $90 / 3773$ & $58 X-7,29$ & Bulk & 1.82 & -0.30 & 576.29 & 18.47 & A91/2791 & $61 X-5,69$ & G. venez. & 1.71 & -1.02 \\
\hline 549.79 & 17.22 & A $91 / 772$ & $58 \times-7,29$ & D. alt. & 2.36 & -1.07 & 577.69 & 18.53 & A90/3118 & $61 X-6,59$ & Bulk & 1.06 & -0.41 \\
\hline 549.79 & 17.22 & A $91 / 773$ & $58 \times-7,29$ & G. venez & 2.01 & -0.52 & 577.69 & 18.53 & A91/2792 & $61 X-6,59$ & D. alt. & 1.70 & -0.84 \\
\hline 549.79 & 17.22 & A91/3219 & $58 \times-7,29$ & Cib. spp. & 0.85 & 1.24 & 577.69 & 18.53 & A91/3023 & $61 \times-6,59$ & Cib. spp. & 0.78 & 1.09 \\
\hline 550.89 & 17.27 & A91/2836 & $59 X-1,69$ & Cib. spp. & 1.62 & 1.12 & 577.69 & 18.53 & A91/2793 & $61 \times-6,59$ & G. venez & 1.36 & -0.43 \\
\hline 550.89 & 17.27 & A $91 / 2651$ & $59 \times-1,69$ & G. venez. & 2.35 & -1.09 & 578.89 & 18.59 & A90/3119 & $61 X-7.29$ & Bulk & 1.04 & -0.07 \\
\hline 550.89 & 17.27 & A91/2650 & $59 X-1,69$ & D. alt. & 2.56 & -1.29 & 578.89 & 18.59 & A91/2795 & $61 X-7,29$ & G. venez. & 1.52 & -0.65 \\
\hline 550.89 & 17.27 & A $90 / 3101$ & $59 X-1,69$ & Bulk & 1.95 & -0.26 & 578.89 & 18.59 & A91/2794 & $61 X-7,29$ & D. alt. & 1.87 & -0.71 \\
\hline 552.29 & 17.34 & A $91 / 2653$ & $59 X-2,59$ & G. venez. & 1.88 & -0.51 & 579.99 & 18.68 & A91/2796 & $62 \mathrm{R}-1,69$ & D. alt. & 1.79 & -0.69 \\
\hline 552.29 & 17.34 & A90/3102 & $59 \times-2,59$ & Bulk & 1.72 & -0.07 & 579.99 & 18.68 & A91/2797 & $62 \mathrm{R}-1,69$ & G. venez & 1.49 & -0.76 \\
\hline 552.29 & 17.34 & A91/2652 & $59 X-2,59$ & D. alt. & 2.06 & -0.72 & 579.99 & 18.68 & A $90 / 3120$ & $62 \mathrm{R}-1,69$ & Bulk & 1.13 & -0.17 \\
\hline 552.29 & 17.34 & A91/2838 & $59 X-2,59$ & Cib. spp. & 1.39 & 0.72 & 581.39 & 18.81 & A $90 / 3121$ & $62 R-2,59$ & Bulk & 0.93 & -0.12 \\
\hline 553.89 & 17.41 & A91/2840 & $59 \times-3,69$ & Cib. spp. & 1.06 & 1.54 & 581.39 & 18.81 & A91/3025 & $62 \mathrm{R}-2,59$ & Cass. spp. & 0.08 & 0.60 \\
\hline 553.89 & 17.41 & A91/2654 & $59 X-3,69$ & D. alt. & 2.10 & -0.69 & 581.39 & 18.81 & A91/2799 & $62 \mathrm{R}-2,59$ & G. venez. & 1.77 & -1.02 \\
\hline 553.89 & 17.41 & A $91 / 2655$ & $59 X-3,69$ & G. venez. & 1.67 & -0.54 & 581.39 & 18.81 & A91/2798 & $62 \mathrm{R}-2,59$ & D. alt. & 1.04 & -0.74 \\
\hline 553.89 & 17.41 & A $90 / 3103$ & $59 \times-3,69$ & Bulk & 1.78 & -0.05 & 582.99 & 18.96 & A91/2801 & $62 \mathrm{R}-3,69$ & G. venez. & 1.39 & -0.41 \\
\hline 555.29 & 17.48 & A91/2656 & $59 \times-4,59$ & D. alt. & 1.75 & -1.29 & 582.99 & 18.96 & A91/3026 & $62 \mathrm{R}-3,69$ & Cib. spp. & 0.88 & 1.48 \\
\hline 555.29 & 17.48 & A91/2657 & $59 X-4,59$ & G. venez. & 2.22 & -1.37 & 582.99 & 18.96 & A91/2800 & $62 \mathrm{R}-3,69$ & D. alt. & 1.71 & -0.84 \\
\hline 555.29 & 17.48 & A $91 / 2843$ & $59 \times-4,59$ & Cib. spp. & 0.81 & 0.70 & 582.99 & 18.96 & A $90 / 3122$ & $62 R-3,69$ & Bulk & 1.24 & -0.09 \\
\hline 555.29 & 17.48 & A $90 / 3104$ & $59 \times-4,59$ & Bulk & 1.45 & -0.40 & 584.39 & 19.09 & A $90 / 3123$ & $62 \mathrm{R}-4,59$ & Bulk & 1.32 & -0.12 \\
\hline 556.89 & 17.56 & A91/2658 & $59 X-5,69$ & D. alt. & 2.15 & -1.18 & 584.39 & 19.09 & A $91 / 2802$ & $62 R-4,59$ & D. alt. & 2.22 & -0.57 \\
\hline 556.89 & 17.56 & A $90 / 3105$ & $59 X-5,69$ & Bulk & 1.57 & -0.10 & 584.39 & 19.09 & A91/2803 & $62 \mathrm{R}-4,59$ & $G$. venez & 1.51 & 0.44 \\
\hline 556.89 & 17.56 & A91/2659 & $59 X-5,69$ & G. venez. & 1.75 & -0.46 & 585.99 & 19.24 & A91/2805 & $62 R-5,69$ & G. venez & 1.41 & -0.15 \\
\hline 558.29 & 17.62 & A $91 / 2661$ & $59 X-6,59$ & G. venez. & 1.41 & -0.84 & 585.99 & 19.24 & A91/3027 & $62 R-5,69$ & Cib. spp. & 0.88 & 1.37 \\
\hline 558.29 & 17.62 & A $91 / 2660$ & $59 \times-6,59$ & D. alt. & 1.52 & -1.01 & 585.99 & 19.24 & A90/3124 & $62 R-5,69$ & Bulk & 1.22 & -0.18 \\
\hline 558.29 & 17.62 & A90/3106 & $59 X-6,59$ & Bulk & 1.38 & -0.18 & 585.99 & 19.24 & A91/2804 & $62 R-5,69$ & D. alt. & 1.42 & -0.68 \\
\hline 559.49 & 17.68 & A $91 / 2770$ & $59 X-7,29$ & D. alt. & 2.02 & -0.65 & 587.39 & 19.37 & A91/2809 & $62 \mathrm{R}-6,59$ & G. venez & 1.37 & -0.45 \\
\hline 559.49 & 17.68 & A $91 / 2771$ & $59 \times-7,29$ & G. venez & 1.56 & -0.71 & 587.39 & 19.37 & A91/2808 & $62 \mathrm{R}-6,59$ & D. alt. & 1.44 & -0.53 \\
\hline 559.49 & 17.68 & A90/3107 & $59 \times-7,29$ & Bulk & 1.45 & -0.13 & 587.39 & 19.37 & A $91 / 2810$ & $62 \mathrm{R}-6,59$ & Cib. spp. & 1.06 & 1.39 \\
\hline 560.59 & 17.73 & A91/2773 & $60 X-1,69$ & G. venez. & 1.64 & -0.45 & 587.39 & 19.37 & A $90 / 3125$ & $62 \mathrm{R}-6,59$ & Bulk & 1.21 & -0.09 \\
\hline 560.59 & 17.73 & A91/2772 & $60 \times-1,69$ & D. alt. & 2.10 & -0.93 & 588.59 & 19.48 & A $90 / 3126$ & $62 R-7,29$ & Bulk & 1.34 & -0.09 \\
\hline 560.59 & 17.73 & A90/3108 & $60 \times-1,69$ & Bulk & 1.65 & -0.10 & 589.69 & 19.54 & A $90 / 3147$ & $63 \mathrm{R}-1,69$ & Bulk & 1.14 & -0.31 \\
\hline 561.99 & 17.80 & A90/3109 & $60 \times-2,59$ & Bulk & 1.49 & -0.19 & 589.69 & 19.54 & A91/2813 & $63 R-1,69$ & Cib. spp. & 0.65 & 1.40 \\
\hline 561.99 & 17.80 & A91/2774 & $60 \times-2,59$ & D. alt. & 1.97 & -0.84 & 589.69 & 19.54 & A $91 / 2812$ & $63 \mathrm{R}-1,69$ & $G$. venez & 1.85 & -0.60 \\
\hline 561.99 & 17.80 & A $91 / 2775$ & $60 \times-2,59$ & G. venez & 1.85 & -0.84 & 589.69 & 19.54 & A91/2811 & $63 R-1,69$ & D. alt. & 2.23 & -0.61 \\
\hline 563.59 & 17.87 & A91/2776 & $60 \times-3,69$ & D. alt. & 2.16 & -0.88 & 591.09 & 19.59 & A $91 / 2814$ & $63 R-2,59$ & D. alt. & 1.47 & -0.75 \\
\hline 563.59 & 17.87 & A $91 / 2777$ & $60 \times-3,69$ & G. venez. & 2.15 & -0.79 & 591.09 & 19.59 & A90/3148 & $63 \mathrm{R}-2,59$ & Bulk & 1.26 & -0.19 \\
\hline 563.59 & 17.87 & $\mathrm{~A} 90 / 3110$ & $60 X-3,69$ & Bulk & 1.54 & -0.08 & 591.09 & 19.59 & A91/2816 & $63 R-2,59$ & Cib. spp. & 0.04 & 1.52 \\
\hline 564.99 & 17.94 & A $91 / 2779$ & $60 \times-4,59$ & G. venez. & 1.60 & -0.65 & 591.09 & 19.59 & A91/2815 & $63 R-2,59$ & G. venez & 1.24 & -0.38 \\
\hline 564.99 & 17.94 & A91/2778 & $60 \times-4,59$ & D. alt. & 2.37 & -0.90 & 592.69 & 19.66 & A91/2819 & $63 R-3,69$ & Cib. spp. & 1.20 & 1.57 \\
\hline 564.99 & 17.94 & A $91 / 3020$ & $60 \times-4,59$ & Cib. spp. & 0.49 & 2.05 & 592.69 & 19.66 & A91/2817 & $63 R-3,69$ & D. alt. & 1.90 & -0.54 \\
\hline 564.99 & 17.94 & A $90 / 3111$ & $60 \times-4,59$ & Bulk & 1.65 & -0.04 & 592.69 & 19.66 & A $91 / 2818$ & $63 R-3,69$ & $G$. venez & 1.50 & -0.06 \\
\hline 566.39 & 18.00 & A90/3112 & $60 X-5,49$ & Bulk & 1.47 & -0.04 & 592.69 & 19.66 & A $90 / 3149$ & $63 \mathrm{R}-3,69$ & Bulk & 1.23 & -0.19 \\
\hline 566.39 & 18.00 & A91/2780 & $60 \times-5,49$ & D. alt. & 2.04 & -0.79 & 594.09 & 19.72 & A $91 / 2822$ & $63 R-4,59$ & Cib. spp. & 0.39 & 1.84 \\
\hline 566.39 & 18.00 & A91/2781 & $60 \times-5,49$ & G. venez. & 1.74 & -0.61 & 594.09 & 19.72 & A $90 / 3150$ & $63 R-4,59$ & Bulk & 1.23 & -0.29 \\
\hline 570.29 & 18.19 & A $91 / 2783$ & $61 X-1,69$ & G. venez. & 1.81 & -0.87 & 594.09 & 19.72 & A91/2821 & $63 R-4,59$ & G. venez & 1.45 & -0.38 \\
\hline 570.29 & 18.19 & A $90 / 3113$ & $61 X-1,69$ & Bulk & 1.69 & 0.66 & 595.69 & 19.78 & A $91 / 2823$ & $63 R-5,69$ & D. alt. & 1.98 & -0.83 \\
\hline 570.29 & 18.19 & A91/2782 & $61 X-1.69$ & D. alt. & 1.77 & -0.54 & 595.69 & 19.78 & A91/2824 & $63 R-5,69$ & G. venez & 1.51 & -0.46 \\
\hline 571.69 & 18.25 & A $91 / 2785$ & $61 X-2,59$ & G. venez. & 1.62 & -0.58 & 595.69 & 19.78 & A $90 / 3151$ & $63 R-5,69$ & Bulk & 1.26 & -0.25 \\
\hline 571.69 & 18.25 & A $91 / 2784$ & $61 X-2,59$ & D. alt. & 1.80 & -0.57 & 595.69 & 19.78 & A $91 / 2825$ & $63 R-5,69$ & $G$-cass. spp. & 0.81 & 1.58 \\
\hline 571.69 & 18.25 & A90/3114 & $61 X-2,59$ & Bulk & 1.29 & -0.24 & 597.09 & 19.84 & A $90 / 3152$ & $63 R-6,59$ & Bulk & 1.31 & -0.36 \\
\hline 573.29 & 18.33 & A90/3115 & $61 X-3,69$ & Bulk & 1.25 & -0.32 & 597.09 & 19.84 & A $91 / 2828$ & $63 R-6,59$ & D. alt. & 1.48 & -0.16 \\
\hline 573.29 & 18.33 & A91/3021 & $61 X-3,69$ & Cass. spp. & 0.42 & 1.49 & 597.09 & 19.84 & A $91 / 2829$ & $63 \mathrm{R}-6,59$ & G. venez & 1.48 & -0.24 \\
\hline 573.29 & 18.33 & A91/2786 & $61 X-3.69$ & D. alt. & 2.14 & -1.08 & 597.09 & 19.84 & A $91 / 2830$ & $63 R-6,59$ & Cib. spp. & 0.77 & 1.73 \\
\hline 573.29 & 18.33 & A91/2787 & $61 X-3,69$ & G. venez. & 2.00 & -0.88 & 598.29 & 19.89 & A $91 / 2832$ & $63 R-7,29$ & D. alt. & 1.99 & -0.97 \\
\hline 574.69 & 18.39 & A91/2789 & $61 X-4,59$ & G. venez & 1.95 & -0.82 & 598.29 & 19.89 & A $91 / 2833$ & $63 R-7,29$ & G. venez. & 1.51 & -0.48 \\
\hline 574.69 & 18.39 & A $91 / 3022$ & $61 X-4,59$ & Cib. spp. & 1.02 & 1.21 & 598.29 & 19.89 & A91/2834 & $63 R-7,29$ & Cib. spp. & 0.83 & 1.32 \\
\hline 574.69 & 18.39 & A91/2788 & $61 X-4,59$ & D. alt. & 1.95 & -1.00 & 598.29 & 19.89 & A $90 / 3153$ & $63 R-7,29$ & Bulk & 1.31 & -0.18 \\
\hline 574.69 & 18.39 & A90/3116 & $61 X-4,59$ & Bulk & 1.36 & -0.33 & & & & & & & \\
\hline
\end{tabular}

Notes: $D$. alt.$=$ Dentoglobigerina altispira, $G$. ven.$=$ Globoquadrina venezuelana, Cib. spp. $=$ Cibicidoides spp., Cib. mun. $=$ Cibicidoides mundulus, $P$. wuell. $=$ Planulina wuellerstorfi, Cass. spp. = Cassidulina spp., and $G$-cass. spp. = Globocassidulina spp. 